\title{
Identification and Pathogenicity of Fungal Species Associated with Canker Diseases of Pistachio in California
}

\author{
Mohamed T. Nouri, ${ }^{1,2}$ Daniel P. Lawrence, ${ }^{2}$ Leslie A. Holland, ${ }^{1,2}$ David A. Doll, ${ }^{3}$ Craig E. Kallsen, ${ }^{4}$ Catherine M. Culumber, \\ and Florent P. Trouillas ${ }^{1,2, \dagger}$ \\ ${ }^{1}$ Kearney Agricultural Research and Extension Center, Parlier, CA 93648 \\ ${ }^{2}$ University of California, Davis, Department of Plant Pathology, Davis, CA 95616 \\ ${ }^{3}$ University of California Cooperative Extension Merced County, Merced, CA 95341 \\ ${ }^{4}$ University of California Cooperative Extension Kern County, Bakersfield, CA 93307 \\ ${ }^{5}$ University of California Cooperative Extension Fresno County, Fresno, CA 93710
}

\begin{abstract}
A survey was conducted during 2015 and 2016 in pistachio orchards throughout the San Joaquin Valley of California to investigate the occurrence of canker diseases and identify the pathogens involved. Cankers and dieback symptoms were observed mainly in orchards aged $>15$ years. Symptoms of canker diseases included brown to dark brown discoloration of vascular tissues, wood necrosis, and branch dieback. In total, 58 fungal isolates were obtained from cankers and identified based on multilocus phylogenetic analyses (internal transcribed spacer, glyceraldehyde 3-phosphate dehydrogenase, $\beta$-tubulin, calmodulin, actin 1 , and translation elongation fac-

pistaciae, Diaporthe ambigua, Didymella glomerata, Diplodia mutila, Neofusicoccum mediterraneum, Phaeoacremonium canadense, and Schizophyllum commune. Pathogenicity tests conducted in the main pistachio cultivars Kerman, Golden Hills, and Lost Hills using the mycelium-plug method indicated that all fungal species were pathogenic to Pistacia vera. All species tested caused cankers in pistachio branches, although virulence among species varied from high to moderate. Overall, N. mediterraneum and Cytospora spp. were the most widespread and virulent species associated with canker diseases of pistachio in California.
\end{abstract} tor $1 \alpha$ ) representing 11 fungal species: Colletotrichum karstii, Cytospora californica, Cytospora joaquinensis, Cytospora parapistaciae, Cytospora
Keywords: fungal pathogens, phylogeny
Cultivated pistachio (Pistacia vera L.), a member of the Anacardiaceae, is native to the eastern Mediterranean and eastern to central Asia (AL-Saghir and Porter 2012). Pistachio is one of the most important commercial nut crops in the world and has been widely cultivated in Iran, Turkey, other Mediterranean countries, and the United States. Commercial production of pistachio nuts began in the late 1970s in California and rapidly expanded to a major commodity in the San Joaquin Valley. By the end of 2017, approximately 100,000 hectares of bearing pistachio trees were present in California, which produced more than $99.1 \%$ (300,138 metric tons) of the crop in the United States (https://www.acpistachios.org). The pistachio industry is relatively new in California, and only a few above-ground diseases have been reported (Michailides 2005). Botryosphaeria panicle and shoot blight caused by several species in the Botryosphaeriaceae, Botrytis blossom and shoot blight caused by Botrytis cinerea Pers., and Alternaria late blight caused by Alternaria alternata (Fr.) Keissl., A. arborescens E.G. Simmons, and A. infectoria E.G. Simmons. are considered major threats to the California pistachio industry (Ashworth et al. 1986; Chen et al. 2014a; Ma et al. 2001; Michailides 2005; Pryor and Michailides 2002).

In recent years, California has been severely affected by unfavorable environmental conditions for pistachio production. Plant stress as a result of water limitation and heat, the general intensification of agricultural practices, aging of pistachio plantings, and

${ }^{\dagger}$ Corresponding author: F. P. Trouillas; flotrouillas@ucdavis.edu

Funding: The California Pistachio Research Board provided funding for this research.

The author(s) declare no conflict of interest.

Accepted for publication 17 April 2019.

(C) 2019 The American Phytopathological Society establishment of new cultivars may contribute to changes in disease incidence and distribution. Surveys conducted in 2015 and 2016 of commercial pistachio orchards detected cankers in the trunks and branches of mature pistachio trees in multiple orchards in California. To date, little is known about the fungi that cause canker diseases in pistachio, although species in the Botryosphaeriaceae have been shown to cause branch cankers at a later stage of Botryosphaeria panicle and shoot blight (Michailides 1991; Michailides et al. 2002). Species in the Botryosphaeriaceae were found to be associated with pistachio branch and scaffold cankers in Australia and Iran, respectively (Mohammadi et al. 2015; Wunderlich et al. 2012). Lasiodiplodia exigua Linald., Deidda \& A.J.L. Phillips (syn. L. americana S.F. Chen, G.Q. Li \& Michailides) and Neofusicoccum hellenicum S.F. Chen, G.Q. Li \& Michailides were isolated from blighted shoots of pistachio in Arizona and Greece, respectively, and were capable of causing lesions on pistachio branches in the field (Chen et al. 2015). Botryosphaeria dothidea (Moug. ex Fr.) Ces. \& De Not. was shown to cause basal cankers of pistachio rootstocks in South Africa (Swart and Blodgett 1998) and shoot blight and cankers of pistachio in Australia (Wunderlich et al. 2012). Mohammadi et al. (2015) indicated that Botryosphaeria dothidea, Dothiorella viticola A.J.L. Phillips \& J. Luque, and N. parvum (Pennycook \& Samuels) Crous, Slippers \& A.J.L. Phillips caused significant wood lesions in cultivated and wild pistachio species in Iran. Linaldeddu et al. (2016) demonstrated the direct involvement of four species in the Botryosphaeriaceae (Diplodia olivarum A.J.L. Phillips, Frisullo \& Lazzizera, Diplodia insularis Linald., A. Alves \& A.J.L. Phillips, N. cryptoaustrale Pavlic, Maleme, Slippers \& M.J. Wingf., and N. luteum (Pennycook \& Samuels) Crous, Slippers \& A.J.L. Phillips) in the etiology of canker and dieback symptoms of Pistacia lentiscus L. in Italy. $N$. australe (Slippers, Crous \& M.J. Wingf.) Crous, Slippers \& A.J.L. Phillips was shown to cause canker disease in Spain (Armengol et al. 2008).

Cytospora species cause cankers and dieback on many genera of hardwoods and coniferous trees, but rarely on herbaceous plants and monocots (Adams et al. 2005). Cytospora canker is a worldwide 
problem and affects more than 85 species of woody shrubs and trees, including important agricultural crops (Adams et al. 2005, 2006; Farr and Rossman 2015; Lawrence et al. 2017a, 2018). In California, a few species of Cytospora were reported to affect sweet cherry (Leucostoma canker) (Trouillas et al. 2012), peach and nectarine (French 1989), and French prune (Bertrand and English 1976). To date,

Table 1. Isolates sequenced and used for phylogenetic study and pathogenicity tests in this study

\begin{tabular}{|c|c|c|c|c|c|c|c|c|}
\hline \multirow[b]{2}{*}{ Species } & \multirow[b]{2}{*}{ Isolate } & \multirow[b]{2}{*}{ Location $^{x}$} & \multicolumn{6}{|c|}{ GenBank accession number ${ }^{y}$} \\
\hline & & & ITS & GAPDH & TUB2 & CAL & TEF-1 $\alpha$ & ACT1 \\
\hline \multirow[t]{2}{*}{ Colletotrichum karstii } & KARE1092z & Fresno & MH837461 & MH837462 & MH891128 & - & - & - \\
\hline & KARE1093z & Fresno & MH837460 & MH837463 & MH891129 & - & - & - \\
\hline \multirow[t]{7}{*}{ Cytospora californica } & KARE264 & Kern & MG971920 & - & MG971780 & - & MG971630 & MG972069 \\
\hline & KARE265 & Kern & MG971914 & - & MG971776 & - & MG971624 & MG972064 \\
\hline & KARE303 & Kern & MG971913 & - & MG971775 & - & MG971623 & MG972063 \\
\hline & KARE324 & Kern & MG971911 & - & MG971773 & - & MG971621 & MG972061 \\
\hline & KARE325 & Kern & MG971918 & - & - & - & MG971628 & MG972067 \\
\hline & KARE326 & Kern & MG971919 & - & - & - & MG971629 & MG972068 \\
\hline & KARE1091z & Kern & MG971946 & - & MG971790 & - & MG971662 & MG972096 \\
\hline \multirow{2}{*}{ Cytospora joaquinensis } & KARE195z & Kern & MG971894 & - & MG971760 & - & MG971604 & MG972043 \\
\hline & KARE231 & Kern & MG971893 & - & MG971759 & - & MG971603 & MG972042 \\
\hline \multirow[t]{4}{*}{ Cytospora parapistaciae } & KARE232 & Kern & MG971807 & - & MG971672 & - & MG971522 & MG971957 \\
\hline & KARE268 & Kern & MG971806 & - & MG971671 & - & MG971521 & MG971956 \\
\hline & KARE269 & Kern & MG971805 & - & MG971670 & - & MG971520 & MG971955 \\
\hline & KARE270 & Kern & MG971804 & - & MG971669 & - & MG971519 & MG971954 \\
\hline \multirow[t]{4}{*}{ Cytospora pistaciae } & KARE441 $1^{\mathrm{z}}$ & Merced & MG971800 & - & MG971665 & - & MG971515 & MG971950 \\
\hline & KARE442 & Merced & MG971803 & - & MG971668 & - & MG971518 & MG971953 \\
\hline & KARE443 & Merced & MG971802 & - & MG971667 & - & MG971517 & MG971952 \\
\hline & KARE444 & Merced & MG971801 & - & MG971666 & - & MG971516 & MG971951 \\
\hline \multirow[t]{6}{*}{ Diaporthe ambigua } & KARE272 & Kern & MH891130 & - & - & MH899709 & MH899703 & - \\
\hline & KARE273 & Kern & MH891131 & - & - & MH899710 & MH899704 & - \\
\hline & KARE274z & Kern & MH891132 & - & - & MH899711 & MH899705 & - \\
\hline & KARE275 & Kern & MH891133 & - & - & MH899712 & MH899706 & - \\
\hline & KARE1103 & Kern & MH891134 & - & - & MH899713 & MH899707 & - \\
\hline & KARE1410 & Kern & MH891135 & - & - & MH899714 & MH899708 & - \\
\hline \multirow[t]{9}{*}{ Didymella glomerata } & KARE189 & Kern & MH899715 & - & MH899733 & - & MH899724 & - \\
\hline & KARE315 & Merced & MH899716 & - & MH899734 & - & MH899725 & - \\
\hline & KARE456 & Fresno & MH899717 & - & MH899735 & - & MH899726 & - \\
\hline & KARE457 & Fresno & MH899718 & - & MH899736 & - & MH899727 & - \\
\hline & KARE466 & Fresno & MH899719 & - & MH899737 & - & MH899728 & - \\
\hline & KARE467 & Fresno & MH899720 & - & MH899738 & - & MH899729 & - \\
\hline & KARE468z & Fresno & MH899721 & - & MH899739 & - & MH899730 & - \\
\hline & KARE469 & Fresno & MH899722 & - & MH899740 & - & MH899731 & - \\
\hline & KARE470 & Fresno & MH899723 & - & MH899741 & - & MH899732 & - \\
\hline \multirow{3}{*}{ Diplodia mutila } & KARE1195 & Glenn & MH899742 & - & MH922944 & - & MH899745 & - \\
\hline & KARE1846 & Madera & MH899743 & - & MH922945 & - & MH899746 & - \\
\hline & KARE1849 & Madera & MH899744 & - & MH922946 & - & MH899747 & - \\
\hline \multirow[t]{15}{*}{ Neofusicoccum mediterraneum } & KARE350 & Merced & MH919427 & - & MH919457 & - & MH919442 & - \\
\hline & KARE351 & Merced & MH919428 & - & MH919458 & - & MH919443 & - \\
\hline & KARE353 & Merced & MH919429 & - & MH919459 & - & MH919444 & - \\
\hline & KARE354 & Merced & MH919430 & - & MH919460 & - & MH919445 & - \\
\hline & KARE430 & Merced & МH919431 & - & MH919461 & - & MH919446 & - \\
\hline & KARE432 & Merced & MH919432 & - & MH919462 & - & MH919447 & - \\
\hline & KARE445 & Merced & МH919433 & - & MH919463 & - & MH919448 & - \\
\hline & KARE1372 & Fresno & MH919434 & - & MH919464 & - & MH919449 & - \\
\hline & KARE1373 & Fresno & MH919435 & - & MH919465 & - & MH919450 & - \\
\hline & KARE1842 & Madera & МH919436 & - & MH919466 & - & MH919451 & - \\
\hline & KARE1843 & Madera & MH919437 & - & MH919467 & - & MH919452 & - \\
\hline & KARE1844 & Madera & MH919438 & - & MH919468 & - & MH919453 & - \\
\hline & KARE1845 & Madera & MH919439 & - & MH919469 & - & MH919454 & - \\
\hline & KARE1847 & Madera & MH919440 & - & MH919470 & - & MH919455 & - \\
\hline & KARE1848 & Madera & МH919441 & - & MH919471 & - & MH919456 & - \\
\hline \multirow[t]{3}{*}{ Phaeoacremonium canadense } & KARE434 ${ }^{z}$ & Merced & MH922938 & - & MH931005 & - & MH922941 & - \\
\hline & KARE435 & Merced & MH922939 & - & MH931006 & - & MH922942 & - \\
\hline & KARE436 & Merced & MH922940 & - & MH931007 & - & MH922943 & - \\
\hline \multirow[t]{3}{*}{ Schizophyllum commune } & KARE438 & Merced & MH931008 & - & - & - & - & - \\
\hline & KARE439z & Merced & MH931009 & - & - & - & - & - \\
\hline & KARE $440^{\mathrm{z}}$ & Merced & MH931010 & - & - & - & - & - \\
\hline
\end{tabular}

${ }^{\mathrm{x}}$ Counties in California.

y ITS $=$ internal transcribed spacer, GAPDH $=$ glyceraldehyde 3 -phosphate dehydrogenase, TUB2 $=\beta$-tubulin, CAL $=$ calmodulin, TEF- $1 \alpha=$ translation elongation factor $1 \alpha$, and ACT1 = actin. Dashes indicate that the corresponding gene sequence was not obtained in this study.

${ }^{\mathrm{z}}$ Isolates used in field pathogenicity tests on Pistacia vera in this study. KARE $=$ Kearney Agricultural Research and Extension Center. 
approximately 627 Cytospora species have been described according to Index Fungorum; however, the occurrence of these species in pistachio orchards has not yet been thoroughly investigated. Lawrence et al. (2018) described 10 new species and one new combination of Cytospora isolated from diverse declining fruit and nut crops in California, including five species associated with pistachio canker and dieback diseases. Swart and Blodgett (1998) reported an unidentified species of Cytospora to be associated with basal canker of Pistacia atlantica Desf. and Pistacia integerrima J.L. Stewart ex Brandis rootstocks in South Africa. However, this species was not considered pathogenic in this study and the authors suggested that it may be a secondary colonizer of pistachio rootstock tissues.

Phaeoacremonium spp. are known to exist as endophytes (Mostert et al. 2005) or as pathogens causing dieback or decline of various woody hosts worldwide (Mostert et al. 2006), including many important agricultural fruit and nut crops such as almond (Gramaje et al. 2012), apricot (Damm et al. 2008), and grapes (Gramaje et al. 2015; Mostert et al. 2005). Mohammadi et al. (2015) reported the occurrence and pathogenicity of four Phaeoacremonium spp. from symptomatic cultivated and wild pistachio trees, with Phaeoacremonium parasiticum (Ajello, Georg \& C.J.K. Wang) W. Gams, Crous \& M.J. Wingf. being the most aggressive and prevalent species in Iran. Liberomyces pistaciae Voglmayr, S. Vitale, D. Aiello, Guarnaccia, Luongo \& Belisario was identified as a causal agent of pistachio cankers and decline in Italy (Vitale et al. 2018).

During 2015 and 2016, several diseased pistachio samples showing branch cankers as well as dieback were collected or submitted to our cooperative extension laboratory for disease diagnosis. Although several putative fungal pathogens were isolated from these cankers, little is known about the etiology, biology, and distribution of canker diseases affecting pistachio trees in California. Therefore, the objectives of this study were (i) to identify fungal species associated with pistachio canker diseases using molecular methods and (ii) to determine the pathogenicity on Pistacia vera of various fungal species isolated from pistachio cankers in California.

\section{Materials and Methods}

Field surveys and fungal isolations. Field observations were conducted in 2015 and 2016 in the main pistachio-producing counties of the San Joaquin Valley in California to investigate the occurrence of canker diseases. Plant samples with cankers or dieback symptoms were collected for detailed examination and fungal isolations. Small wood pieces $(3 \times 3 \times 3 \mathrm{~mm})$ from the margins of necrotic and apparently healthy tissues were surface disinfected in $0.5 \%$ sodium hypochlorite solution for $2 \mathrm{~min}$ and subsequently rinsed twice with sterile water. After drying on sterile paper towels, the sterilized segments were plated onto acidified potato dextrose agar (APDA; $2.6 \mathrm{ml}$ of $25 \%$ [vol/vol] lactic acid per liter of medium) and incubated in the laboratory at $25^{\circ} \mathrm{C}$ with approximately $12 \mathrm{~h}$ of daylight and $12 \mathrm{~h}$ of darkness. Consistent fungal colonies that emerged from the diseased tissues were excised using a sterile transfer needle and cultured on APDA. Pure fungal cultures were obtained by transferring single hyphal tips or single spores to potato dextrose agar (PDA) and cultures were incubated at $25 \pm 1^{\circ} \mathrm{C}$ for 5 to 7 days until fungal colonies were large enough to be examined. All isolates collected for this study are detailed in Table 1 and are maintained in the culture collection of the Department of Plant Pathology of the University of California, Davis, at the Kearney Agricultural Research and Extension Center (KARE) in Parlier, California.

DNA extraction, PCR amplification, sequencing, and phylogenetic analyses. Total genomic DNA was extracted from approximately 7-day-old PDA colonies by lightly scraping the mycelium with a sterile scalpel and following the manufacturer's protocol for the FastDNA Spin Kit (MP Biomedicals, Burlingame, CA). The primer pairs ITS1/ITS4 (White et al. 1990), Bt2a/Bt2b (Glass and Donaldson 1995), and T1 (O'Donnell and Cigelnik 1997) and Bt2b (Glass and Donaldson 1995), EF1-728F/EF1986R (Carbone and Kohn 1999), ACT-512F/ACT-783R (Carbone and Kohn 1999), CAL-228F/CAL-737R (Carbone and Kohn
1999), and GDF1/GDR1 (Guerber et al. 2003) were used to amplify the nuclear rDNA internal transcribed spacer (ITS) region including ITS1, the intervening 5.8S rRNA gene and ITS2, and portions of $\beta$-tubulin (TUB2), translation elongation factor $1-\alpha$ (TEF1), actin (ACT1), calmodulin (CAL), and glyceraldehyde 3-phosphate dehydrogenase (GAPDH), respectively. Amplification products were visually checked by electrophoresis $(120 \mathrm{~V}$ for $25 \mathrm{~min})$ in a $1.5 \%$ agarose gel for presence and size followed by purification using ExoSap-IT Product Cleanup Reagent (Affymetrix, Santa Clara, CA). PCR amplicons were sequenced in both directions using an ABI 3730 Capillary Electrophoresis Genetic Analyzer (College of Biological Sciences Sequencing Facility, University of California, Davis, CA).

DNA chromatograms were edited with ContigExpress Vector NTI Advance 10 (Invitrogen, Carlsbad, CA), and consensus sequences (Table 1) were subjected to BLASTn queries in NCBI and the curated database TrunkDiseaseID.org (Lawrence et al. 2017b) to obtain preliminary identifications and sequences with high similarity to ex-type or representative specimens for phylogenetic reference (Table 2). Multiple sequence alignments were conducted in MEGA version 6 (Tamura et al. 2013) and manually adjusted where necessary in Mesquite version 3.10 (Maddison and Maddison 2016). Alignments were submitted to TreeBASE under the accession number S22789. Phylogenetic analyses were performed in MEGA version 6 with two different optimality search criteria, maximum likelihood (ML) and maximum parsimony (MP), on nine separate data sets of the fungal classes Dothideomycetes (ITS, TUB2, and TEF1) and Sordariomycetes (ITS, TUB2, TEF1, ACT1, CAL, and GAPDH). All resulting tree topologies (data not shown) were visually compared for congruence to combine three Dothideomycetes data sets (ITS + TUB2 + TEF1) and six Sordariomycetes data sets (ITS + TUB2 + TEF1 + ACT1 + CAL + GAPDH) for multigene analyses. For ML analyses, MEGA was used to infer the best model of nucleotide substitution for each data set using the Akaike information criterion (AIC). Each ML analysis used the nearest-neighbor-interchange heuristic search method and clade support was estimated by 1,000 bootstrap replicates. MP analyses consisted of heuristic searches with 1,000 random sequence additions implemented with the tree-bisection-reconnection algorithm, with gaps treated as missing data. Bootstrap analyses were performed as above. Sequences of the basidiomycete Schizophyllum Fr. (Agaricales, Schizophyllaceae) served as the outgroup taxon in all analyses.

Pathogenicity tests. Four independent studies were carried out on pistachio trees in the field in 2016 and 2017 using mycelial plug inoculations. One or two representative isolates per fungal species were selected to evaluate the ability of the various fungal species to cause canker in pistachio. The virulence of the various isolates was also compared by assessing lesion size.

In all four experiments, 19 isolates representing the three fungal classes Agaricomycetes, Dothideomycetes, and Sordariomycetes were used for inoculation. These included Cytospora parapistaciae (KARE270), Cytospora pistaciae (KARE441), Cytospora joaquinensis (KARE195 and KARE231), Cytospora californica (KARE265, KARE325, and KARE1091), Colletotrichum karstii (KARE1092 and KARE1093), Diaporthe ambigua (KARE274 and KARE275), N. mediterraneum (KARE350 and KARE445), Phaeoacremonium canadense (KARE434 and KARE435), S. commune (KARE439 and KARE440), and Didymella glomerata (KARE315 and KARE468) (Table 1).

Pathogenicity tests were conducted in 2016 on mature 15-year-old pistachio trees located at KARE. Inoculations were carried out in the beginning of June in the most widely grown cultivar Kerman. Three additional tests were conducted in June 2017 using 10-year-old Golden Hills, Lost Hills, and Kerman pistachio trees at KARE. For each fungal isolate, one 2- to 3-year-old branch on each of eight trees (eight replicates) was selected randomly. The outer bark at the inoculation area was disinfected by spraying with $70 \%$ ethanol and a 5-mm wound was made using a sterilized cork borer. A 5-mmdiameter mycelium plug from a 7-day-old PDA culture was aseptically inserted into the wound, sealed with petroleum jelly, and then protected with Parafilm. Noncolonized PDA agar plugs served as the negative control. 
Table 2. Isolates and sequences from other studies used in phylogenetic analyses for this study.

\begin{tabular}{|c|c|c|c|c|c|c|c|c|c|}
\hline \multirow[b]{2}{*}{ Species } & \multirow[b]{2}{*}{ Isolate $^{x}$} & \multirow[b]{2}{*}{ Host/substrate } & \multirow[b]{2}{*}{ Location } & \multicolumn{6}{|c|}{ GenBank accession number ${ }^{y}$} \\
\hline & & & & ITS & GAPDH & TUB2 & CAL & TEF-1 $\alpha$ & ACT1 \\
\hline \multirow[t]{2}{*}{ Colletotrichum acutatum } & CBS 111993 & Grevillea $\mathrm{sp}$. & Australia & JQ948349 & JQ948680 & JQ950000 & - & - & - \\
\hline & CBS 112759 & Hakea sericea & South Africa & JQ948391 & JQ948722 & JQ950042 & - & - & - \\
\hline $\begin{array}{l}\text { Colletotrichum } \\
\text { annellatum }\end{array}$ & CBS 129826 & Hevea brasiliensis & Colombia & JQ005222 & JQ005309 & JQ005656 & - & - & - \\
\hline Colletotrichum beeveri & CBS 128527 & $\begin{array}{l}\text { Brachyglottis } \\
\quad \text { repanda }\end{array}$ & New Zealand & JQ005171 & JQ005258 & JQ005605 & - & - & - \\
\hline \multirow[t]{2}{*}{$\begin{array}{l}\text { Colletotrichum } \\
\text { boninense }\end{array}$} & CBS 123755 & $\begin{array}{l}\text { Crinum asiaticum } \\
\text { var. sinicum }\end{array}$ & Japan & JQ005153 & JQ005240 & JQ005588 & - & - & - \\
\hline & CBS 112115 & Leucospermum sp. & Australia & JQ005160 & JQ005247 & JQ005594 & - & - & - \\
\hline $\begin{array}{l}\text { Colletotrichum } \\
\text { constrictum }\end{array}$ & CBS 128504 & $\begin{array}{l}\text { Citrus limon, fruit } \\
\text { rot }\end{array}$ & New Zealand & JQ005238 & JQ005325 & JQ005672 & - & - & - \\
\hline $\begin{array}{c}\text { Colletotrichum } \\
\text { dacrycarpi }\end{array}$ & CBS 130241 & $\begin{array}{l}\text { Dacrycarpus } \\
\text { dacrydioides }\end{array}$ & New Zealand & JQ005236 & JQ005323 & JQ005670 & - & - & - \\
\hline $\begin{array}{l}\text { Colletotrichum } \\
\text { gloeosporioides }\end{array}$ & CBS 112999 & Citrus sinensis & Italy & JQ005152 & JQ005239 & JQ005587 & - & - & - \\
\hline \multirow[t]{2}{*}{ Colletotrichum karstii } & CBS 129830 & $\begin{array}{l}\text { Eucalyptus } \\
\text { grandis }\end{array}$ & South Africa & JQ005200 & JQ005287 & JQ005634 & - & - & - \\
\hline & CBS 113087 & Malus sp. & U.S.A. & JQ005181 & JQ005268 & JQ005615 & - & - & - \\
\hline $\begin{array}{c}\text { Colletotrichum } \\
\text { parsonsiae }\end{array}$ & CBS 128525 & $\begin{array}{l}\text { Parsonsia } \\
\text { capsularis }\end{array}$ & New Zealand & JQ005233 & JQ005320 & JQ005667 & - & - & - \\
\hline \multirow[t]{2}{*}{ Botryosphaeria dothidea } & CMW8000 & Prunus sp. & Switzerland & AY236949 & - & AY236927 & - & AY236898 & - \\
\hline & CMW7780 & Fraxinus excelsior & Switzerland & AY236947 & - & AY236925 & - & AY236896 & - \\
\hline \multirow[t]{2}{*}{ Diplodia mutila } & CBS 112554 & Pyrus communis & Portugal & AY259095 & - & DQ458851 & - & DQ458870 & - \\
\hline & CBS 230.30 & $\begin{array}{l}\text { Phoenix } \\
\text { dactylifera }\end{array}$ & $\begin{array}{l}\text { California, } \\
\text { U.S.A. }\end{array}$ & DQ458886 & - & DQ458849 & - & DQ458869 & - \\
\hline \multirow[t]{2}{*}{ Dothiorella iberica } & CBS 115041 & Quercus ilex & Spain & AY573202 & - & EU673096 & - & AY573222 & - \\
\hline & CBS 113188 & Q. suber & Spain & AY573198 & - & EU673097 & - & EU673278 & - \\
\hline \multirow[t]{2}{*}{ Diplodia seriata } & CBS 112555 & Vitis vinifera & Portugal & AY259094 & - & DQ458856 & - & AY573220 & - \\
\hline & CBS 119049 & Vitis sp. & Italy & DQ458889 & - & DQ458857 & - & DQ458874 & - \\
\hline \multirow[t]{2}{*}{$\begin{array}{c}\text { Lasiodiplodia } \\
\text { theobromae }\end{array}$} & CBS 164.96 & $\begin{array}{l}\text { Fruit on coral reef } \\
\text { coast }\end{array}$ & New Guinea & AY640255 & - & EU673110 & - & AY640258 & - \\
\hline & CBS 124.13 & $\mathrm{~N} / \mathrm{A}^{\mathrm{z}}$ & U.S.A. & DQ458890 & - & DQ458858 & - & DQ458875 & - \\
\hline Neofusicoccum luteum & CBS 110299 & $V$. vinifera & Portugal & AY928043 & - & DQ458848 & - & AY573217 & - \\
\hline \multirow[t]{2}{*}{ N. mediterraneum } & CBS 121718 & Eucalyptus sp. & Greece & GU251176 & - & GU251836 & - & GU251308 & - \\
\hline & CBS 121558 & $V$. vinifera & $\begin{array}{l}\text { California, } \\
\text { U.S.A. }\end{array}$ & GU799463 & - & GU799461 & - & GU799462 & - \\
\hline \multirow[t]{2}{*}{ N. parvum } & CMW9081 & Populus nigra & New Zealand & AY236943 & - & AY236917 & - & AY236888 & - \\
\hline & CMW9080 & Populus nigra & New Zealand & AY236942 & - & AY236916 & - & AY236887 & - \\
\hline \multirow[t]{2}{*}{ N. ribis } & CMW7772 & Ribes sp. & $\begin{array}{l}\text { New York, } \\
\text { U.S.A. }\end{array}$ & AY236935 & - & GU251786 & - & AY236877 & - \\
\hline & CMW7773 & Ribes sp. & $\begin{array}{l}\text { New York, } \\
\text { U.S.A. }\end{array}$ & AY236936 & - & AY236907 & - & AY236878 & - \\
\hline $\begin{array}{l}\text { Diaporthe } \\
\text { australafricana }\end{array}$ & CBS 111886 & V. vinifera & Australia & KC343038 & - & - & KC343280 & KC343764 & - \\
\hline Diaporthe acaciigena & CBS 129521 & Acacia retinodes & Australia & KC343005 & - & - & KC 343247 & KC343731 & - \\
\hline Diaporthe amygdali & CBS 126679 & Prunus dulcis & Portugal & KC343022 & - & - & KC343264 & KC343748 & - \\
\hline Diaporthe eres & CBS 287.74 & Sorbus aucuparia & Netherlands & KC343084 & - & - & KC343326 & KC 343810 & - \\
\hline Diaporthe chamaeropis & CBS 454.81 & $\begin{array}{l}\text { Chamaerops } \\
\text { humilis }\end{array}$ & Greece & KC343048 & - & - & KC343290 & KC343774 & - \\
\hline Diaporthe novem & CBS 127270 & Glycine max & Croatia & KC343156 & - & - & KC343398 & KC343882 & - \\
\hline Diaporthe ambigua & CBS 114015 & Pyrus communis & South Africa & KC343010 & - & - & KC 343252 & KC 343736 & - \\
\hline $\begin{array}{l}\text { Phaeoacremonium } \\
\text { armeniacum }\end{array}$ & ICMP 17421 & $V$. vinifera & New Zealand & EU770224 & - & EU596526 & - & KF764615 & - \\
\hline $\begin{array}{l}\text { Phaeoacremonium } \\
\text { canadense }\end{array}$ & PARC327 & $V$. vinifera & $\begin{array}{l}\text { California, } \\
\text { U.S.A. }\end{array}$ & KF764527 & - & KF764652 & - & KF764581 & - \\
\hline $\begin{array}{l}\text { Phaeoacremonium } \\
\text { fraxinopennsylvanicum }\end{array}$ & CBS 101585 & $V$. vinifera & $\begin{array}{l}\text { California, } \\
\text { U.S.A. }\end{array}$ & AF295328 & - & KF764684 & - & KF764640 & - \\
\hline $\begin{array}{l}\text { Phaeoacremonium } \\
\text { alvesii }\end{array}$ & CBS 110034 & Homo sapiens & Brazil & KF764558 & - & AY579301 & - & KF764612 & - \\
\hline $\begin{array}{l}\text { Phaeoacremonium } \\
\text { australiense }\end{array}$ & CBS 113589 & $V$. vinifera & Australia & KF764560 & - & AY579296 & - & KF764616 & - \\
\hline $\begin{array}{l}\text { Phaeoacremonium } \\
\text { hispanicum }\end{array}$ & CBS 123910 & $V$. vinifera & Spain & KF764563 & - & FJ517164 & - & KF764622 & - \\
\hline & & & & & & & & (Continued & ext paq \\
\hline
\end{tabular}

$\mathrm{x}$ Isolate numbers in bold represent ex-type specimens.

${ }^{\text {y }}$ ITS = internal transcribed spacer, GAPDH = glyceraldehyde 3-phosphate dehydrogenase,TUB2 $=\beta$-tubulin, CAL $=$ calmodulin, TEF-1 $\alpha=$ translation elongation factor $1 \alpha$, and ACT1 = actin. Dashes indicate the corresponding gene sequence was not avalaible from GenBank or was not used in the presnt study. ${ }^{\mathrm{z}} \mathrm{N} / \mathrm{A}=$ not available. 
In all experiments, inoculated and control branches were collected and returned to the laboratory 6 months after inoculations. After the bark was removed, the length of wood discoloration (LWD) was measured upward and downward from the point of inoculation using a digital caliper. Ten pieces $(2 \times 2 \times 5 \mathrm{~mm})$ of necrotic tissue from the edge of each lesion were surface disinfected in $0.5 \%$ sodium hypochlorite solution for $2 \mathrm{~min}$, rinsed twice with sterile water, and plated on APDA to recover the inoculated fungi and complete Koch's postulates. Fungal identity was verified by morphological comparison with the original isolates. The lesion-length data satisfied the normality and homogeneity of variance requirements of analysis of variance (ANOVA) according to the Shapiro-Wilk and Bartlett tests, respectively. ANOVAs were performed in Statistix 10 (Analytical Software, Tallahassee, FL) to evaluate differences in the LWD between the control and fungal treatments. Pairwise mean differences compared with the controls were analyzed with the Dunnett test $(P>$ 0.05).

\section{Results}

Field surveys and fungal isolations. Canker disease symptoms (Fig. 1) were observed in 20 orchards in Glenn, Merced, Madera, Fresno, and Kern counties (Fig. 2). Cankers were mainly observed on pistachio trees aged 15 years and older but only occasionally in younger orchards. Common symptoms included brown to dark brown discoloration of vascular tissues, wood necrosis, and branch dieback (Fig. 1). Branch cankers were found to develop mostly from blighted shoots, pruning wounds, sunburn lesions, and other wounds.
Larger cankers had slightly sunken or flattened areas of bark and cankered cambium, phloem, and sapwood revealed dark discoloration (Fig. 1).

Based on colony morphology (Fig. 3) and phylogenetic analyses (see below), 58 isolates were identified. Cytospora spp. and $N$. mediterraneum were the most common fungi isolated from cankers in mature trees, whereas the other species occurred less frequently. Cytospora spp. accounted for $29.3 \%$ of all isolates, followed by $N$. mediterraneum with $25.9 \%$. The remaining isolates were identified as Didymella glomerata (Corda) Q. Chen \& L. Cai (15.5\%); Diaporthe ambigua Nitschke (10.3\%); Phaeoacremonium canadense Úrbez-Torr., P. Haag \& O'Gorman, S. commune Fr., and Diplodia mutila (Fr.) Mont. (each species with 5.2\%); and Colletotrichum karstii You L. Yang, Zuo Y. Liu, K.D. Hyde \& L. Cai (3.4\%) (Table 1).

Phylogenetic analyses. For ML analyses, the best-fit model of nucleotide substitution was deduced based on the AIC (T92+G for Dothideomycetes and TN93+G+I for Sordariomycetes multigene analyses). Alignment of 62 Dothideomycetes sequences (including the outgroup $S$. commune) resulted in a 1,333-character data set (629 characters were constant, 652 characters were parsimony informative, and 52 characters were parsimony uninformative). MP analysis produced nine equally most parsimonious trees of 1,282 steps and a consistency index (CI), retention index (RI), and rescaled consistency index (RC) of $0.7790,0.9661$, and 0.7581 , respectively. ML and MP analyses of the Dothideomycetes data set revealed that 15 isolates clustered strongly (MP and ML bootstrap values of 99/ $99 \%$, respectively) with the type specimen of $N$. mediterraneum

Table 2. (Continued from previous page)

\begin{tabular}{|c|c|c|c|c|c|c|c|c|c|}
\hline \multirow[b]{2}{*}{ Species } & \multirow[b]{2}{*}{ Isolate $^{x}$} & \multirow[b]{2}{*}{ Host/substrate } & \multirow[b]{2}{*}{ Location } & \multicolumn{6}{|c|}{ GenBank accession number ${ }^{y}$} \\
\hline & & & & ITS & GAPDH & TUB2 & CAL & TEF-1 $\alpha$ & ACT1 \\
\hline $\begin{array}{l}\text { Phaeoacremonium } \\
\text { angustius }\end{array}$ & CBS 249.95 & V. vinifera & $\begin{array}{l}\text { California, } \\
\text { U.S.A. }\end{array}$ & AF197974 & - & AF246814 & - & KF764614 & - \\
\hline $\begin{array}{l}\text { Phaeoacremonium } \\
\text { theobromatis }\end{array}$ & CBS 111586 & Theobroma gileri & Ecuador & KF764567 & - & DQ173106 & - & KF764633 & - \\
\hline $\begin{array}{l}\text { Phaeoacremonium } \\
\text { pallidum }\end{array}$ & CBS 120862 & Prunus armeniaca & South Africa & EU128053 & - & EU128103 & - & KF764627 & - \\
\hline Воегетіа ехіgиа & D 075 & Glycine $\max$ & Poland & EU555533 & - & EU541421 & - & EU543982 & - \\
\hline Epicoccum plurivorum & D 072 & Medicago sativa & Australia & EU573018 & - & EU552932 & - & EU552929 & - \\
\hline E. plurivorum & ICMP 6875 & $\begin{array}{l}\text { Pennisetum } \\
\text { clandestinum }\end{array}$ & New Zealand & EU573019 & - & EU552931 & - & EU552930 & - \\
\hline Didymella pinodella & $\mathrm{D} / 035$ & Glycine max & Hungary & EU573015 & - & EU541416 & - & EU543973 & - \\
\hline Phoma sojicola & $\mathrm{D} / 054$ & Glycine $\max$ & Hungary & EU573023 & - & EU541434 & - & EU543974 & - \\
\hline Didymella pinodella & $\mathrm{D} / 045$ & Hordeum vulgare & Hungary & EU573025 & - & EU541417 & - & EU543971 & - \\
\hline Didymella aurea & CBS 269.93 & M. polymorpha & New Zealand & GU237818 & - & GU237557 & - & - & - \\
\hline \multirow[t]{5}{*}{ Didymella glomerata } & ICMP 15788 & Yисса sp. & New Zealand & EU573017 & - & EU541426 & - & EU543968 & - \\
\hline & CBS 133.72 & $\begin{array}{l}\text { Church wall- } \\
\text { fresco }\end{array}$ & Romania & FJ427004 & - & FJ427115 & - & - & - \\
\hline & CBS 528.66 & $\begin{array}{l}\text { Chrysanthemum } \\
\text { sp. }\end{array}$ & Netherlands & FJ427013 & - & FJ427124 & - & - & - \\
\hline & 15157 & Pistacia vera & $\begin{array}{l}\text { Arizona, } \\
\text { U.S.A. }\end{array}$ & MG570147 & - & MG570145 & - & MG570146 & - \\
\hline & D 034 & Glycine max & Hungary & EU573016 & - & EU541424 & - & EU543969 & - \\
\hline Cytospora parapistaciae & CBS 144506 & Pistacia vera & $\begin{array}{l}\text { California, } \\
\text { U.S.A. }\end{array}$ & MG971804 & - & MG971669 & - & MG971519 & MG971954 \\
\hline Cytospora pistaciae & CBS 144238 & Pistacia vera & $\begin{array}{l}\text { California, } \\
\text { U.S.A. }\end{array}$ & MG971802 & - & MG971667 & - & MG971517 & MG971952 \\
\hline Cytospora joaquinensis & CBS 144235 & Pistacia vera & $\begin{array}{l}\text { California, } \\
\text { U.S.A. }\end{array}$ & MG971895 & - & MG971761 & - & MG971605 & MG972044 \\
\hline Cytospora californica & CBS 144234 & Pistacia vera & $\begin{array}{l}\text { California, } \\
\text { U.S.A. }\end{array}$ & MG971935 & - & - & - & MG971645 & MG972083 \\
\hline $\begin{array}{l}\text { Schizophyllum } \\
\text { umbrinum }\end{array}$ & FL02.1 & N/A & $\begin{array}{l}\text { Florida, } \\
\text { U.S.A. }\end{array}$ & AF249391 & - & - & - & - & - \\
\hline Schizophyllum radiatum & CBS 301.32 & N/A & Panama & AY571060 & - & - & - & - & - \\
\hline Schizophyllum commune & CBS 123304 & H. sapiens & India & LT217530 & - & - & - & - & - \\
\hline
\end{tabular}



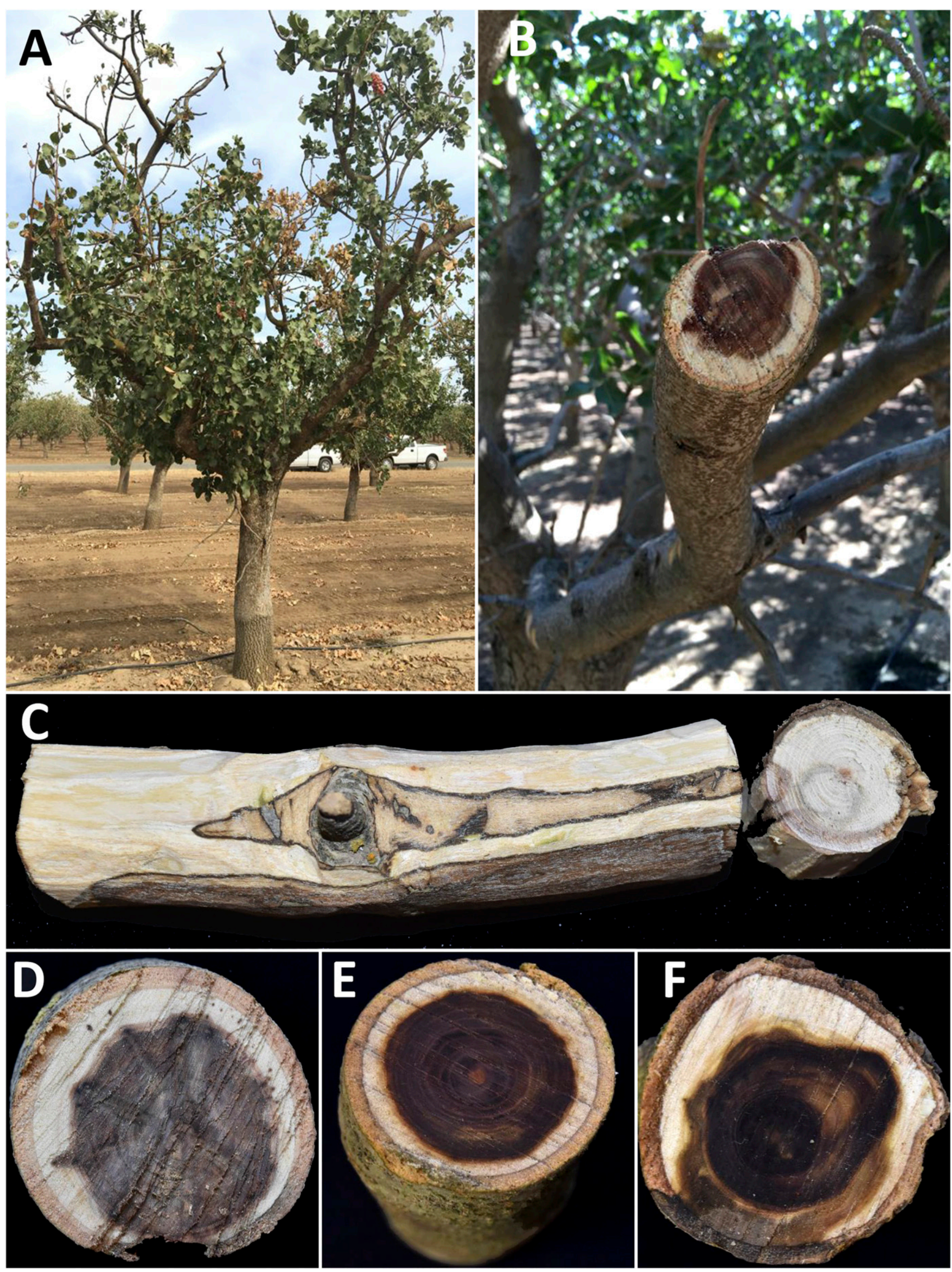

Fig. 1. Canker disease symptoms observed in pistachio orchards in California. A, Branch dieback. B, Canker with vascular discoloration. C, Branch canker developing from a shoot affected by Neofusicoccum mediterraneum. D, Cross-section of a branch canker revealing wood discoloration caused by N. mediterraneum. E and F, Cross-section of branches showing brown to dark brown discoloration of vascular tissues caused by Cytospora spp. 
(CBS 121718); three isolates were identified as Diplodia mutila (100/99\%); nine isolates clustered strongly (99/90\%) with Didymella glomerata, including an isolate that was reported to cause pistachio leaf blight (Moral et al. 2018); and three isolates clustered with $S$. commune with moderate to no support $(82 /<70 \%)$ (Fig. 4).

Alignment of 65 Sordariomycetes sequences (including S. commune) resulted in a 3,329-character data set $(1,599$ characters were constant, 1,414 characters were parsimony informative, and 316

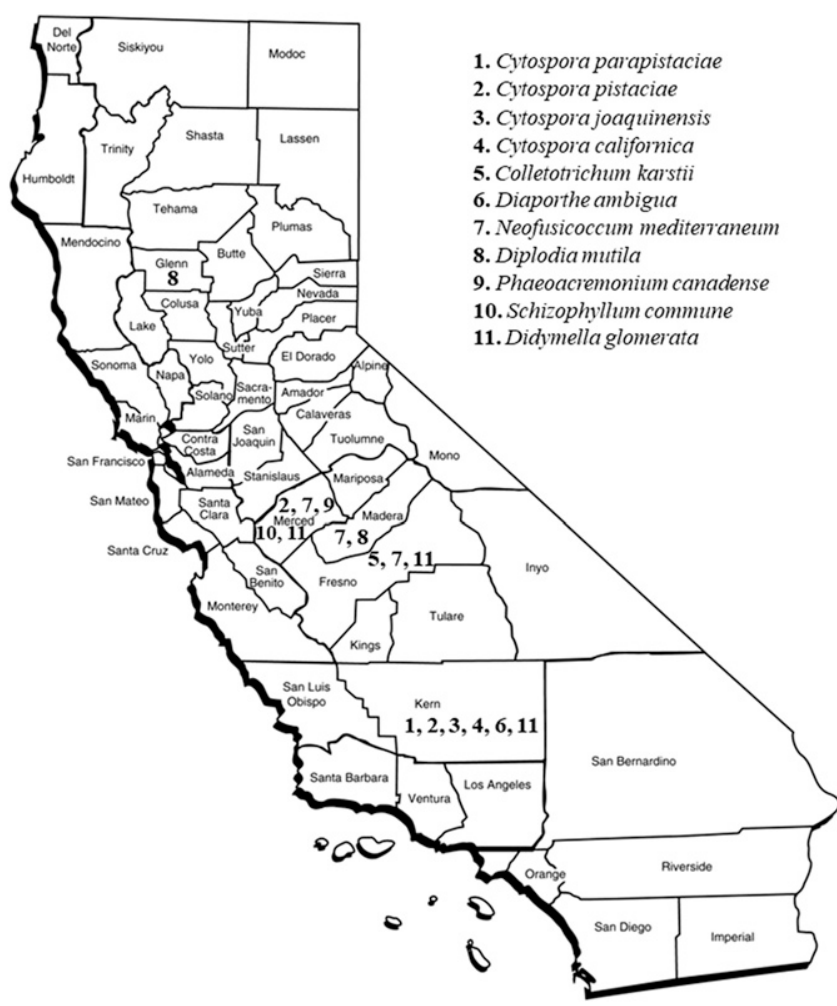

Fig. 2. California map indicating the fungal species detected in each county. The 11 fungal species are indicated as follows: 1, Cytospora parapistaciae; 2, Cytospora pistaciae; 3, Cytospora joaquinensis; 4, Cytospora californica; 5, Colletotrichum karstii; 6, Diaporthe ambigua; 7. Neofusicoccum mediterraneum; 8, Diplodia mutila; 9, Phaeoacremonium canadense; 10, Schizophyllum commune; and 11, Didymella glomerata. characters were parsimony uninformative). MP analysis produced eight equally most parsimonious trees of 3,649 steps and a CI, RI, and RC of $0.6964,0.9186$, and 0.6673 , respectively. ML and MP analyses of the Sordariomycetes data set revealed five fungal genera with 19 Cytospora isolates comprising four strongly supported $(\geq 99$ / $100 \%$ ) species (Cytospora californica D.P. Lawr., L.A. Holland \& Trouillas, Cytospora joaquinensis D.P. Lawr., L.A. Holland \& Trouillas, Cytospora parapistaciae D.P. Lawr., L.A. Holland \& Trouillas, and Cytospora pistaciae D.P. Lawr., L.A. Holland \& Trouillas) (Fig. 5). Six isolates clustered strongly (100/100\%) with sequences of the ex-type of Diaporthe ambigua (CBS 114015). Two isolates clustered (100/90\%) with two representative isolates of Colletotrichum karstii (CBS 113087 and CBS 129830; Damm et al. 2012). Three isolates strongly clustered (99/98\%) with the ex-type specimen of Phaeoacremonium canadense PARC327. Likewise, three isolates were identified as $S$. commune as in the previous analyses.

Pathogenicity tests. In the study conducted in 2016, all fungal species tested produced cankers and/or vascular discoloration in the wood of Kerman pistachio (Fig. 6). Cankers were similar to those observed in naturally infected trees in the field. The average LWD varied among species and isolates, suggesting differences in virulence. However, all isolates tested produced lesions that were significantly longer $(P<0.05)$ compared with the noninoculated control. In this experiment, Cytospora parapistaciae (KARE270) was the most virulent species with canker length averaging $144 \mathrm{~mm}$ after 6 months, followed by $N$. mediterraneum (KARE445) (70 $\mathrm{mm}$ mean length) and Didymella glomerata (KARE315) (69.3 mm mean length). The remaining isolates caused lesions that ranged between 27 and $53 \mathrm{~mm}$ in length (Fig. 7).

In the 2017 experiments, all isolates tested were pathogenic to all three pistachio cultivars used. Isolates KARE350 and KARE445 of $N$. mediterraneum and isolate KARE270 of Cytospora parapistaciae were the most virulent. Mean lesion length varied between 87.8 and $97.2 \mathrm{~mm}$ in Kerman, 102.3 and $104.2 \mathrm{~mm}$ in Lost Hills, and 133.8 and $145.3 \mathrm{~mm}$ in Golden Hills (Figs. 8, 9, and 10, respectively). The remaining fungal species and isolates caused mean lesion lengths ranging from 38.1 to $49.7 \mathrm{~mm}$ for Kerman, 45.3 to $51.3 \mathrm{~mm}$ for Lost Hills, and 40.5 to $56.2 \mathrm{~mm}$ for Golden Hills (Figs. 8, 9, and 10).

All fungi tested in the 2016 and 2017 experiments were recovered from the outer margin of lesions extending from the inoculation points, indicating their ability to colonize the living vascular tissue of pistachio. Fungal recovery varied between 66 and 100\% among isolates. No fungal pathogen was isolated from the controls. ANOVA
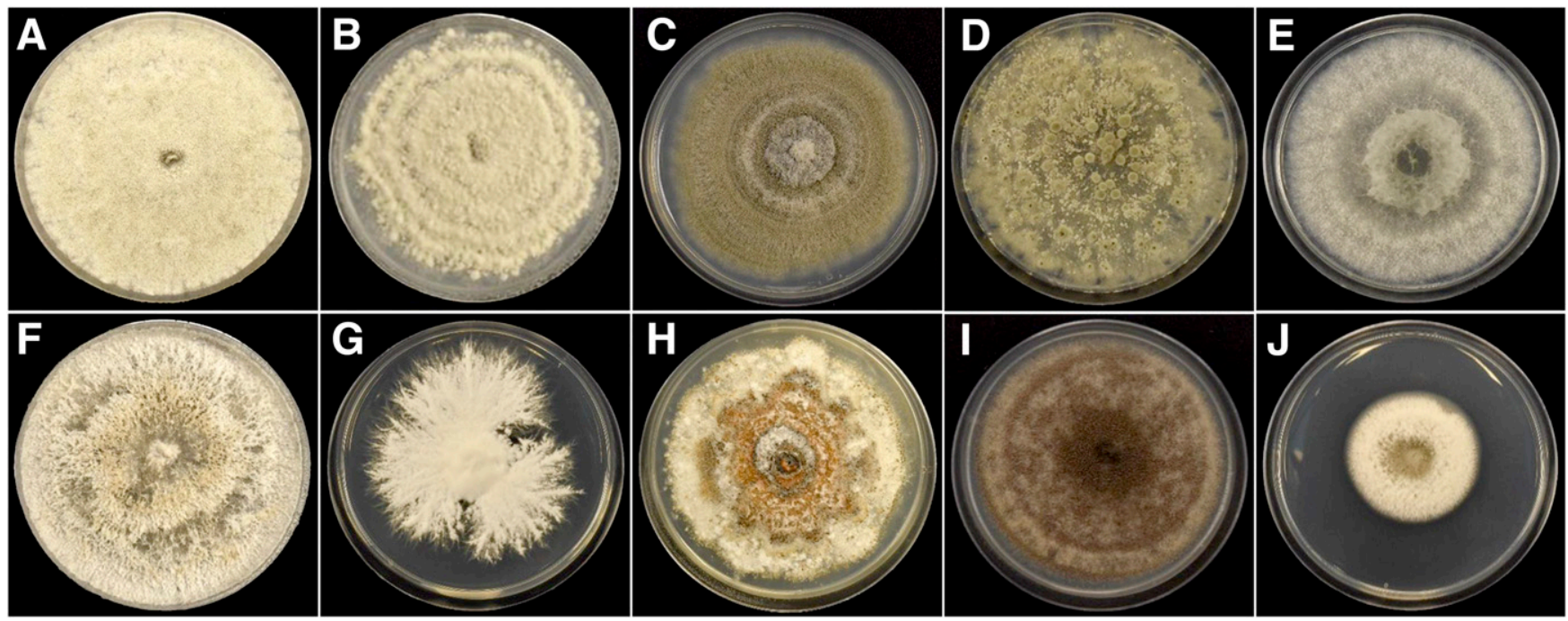

Fig. 3. Colony morphology of fungal species isolated from pistachio trees in California on potato dextrose agar after 12 days of incubation at $25 \pm 1^{\circ} \mathrm{C}$. A, Cytospora joaquinensis. B, Cytospora parapistaciae. C, Cytospora californica. D, Cytospora pistaciae. E, Neofusicoccum mediterraneum. F, Diaporthe ambigua. G, Schizophyllum commune. H, Colletotrichum karstii. I, Didymella glomerata. J, Phaeoacremonium canadense. 


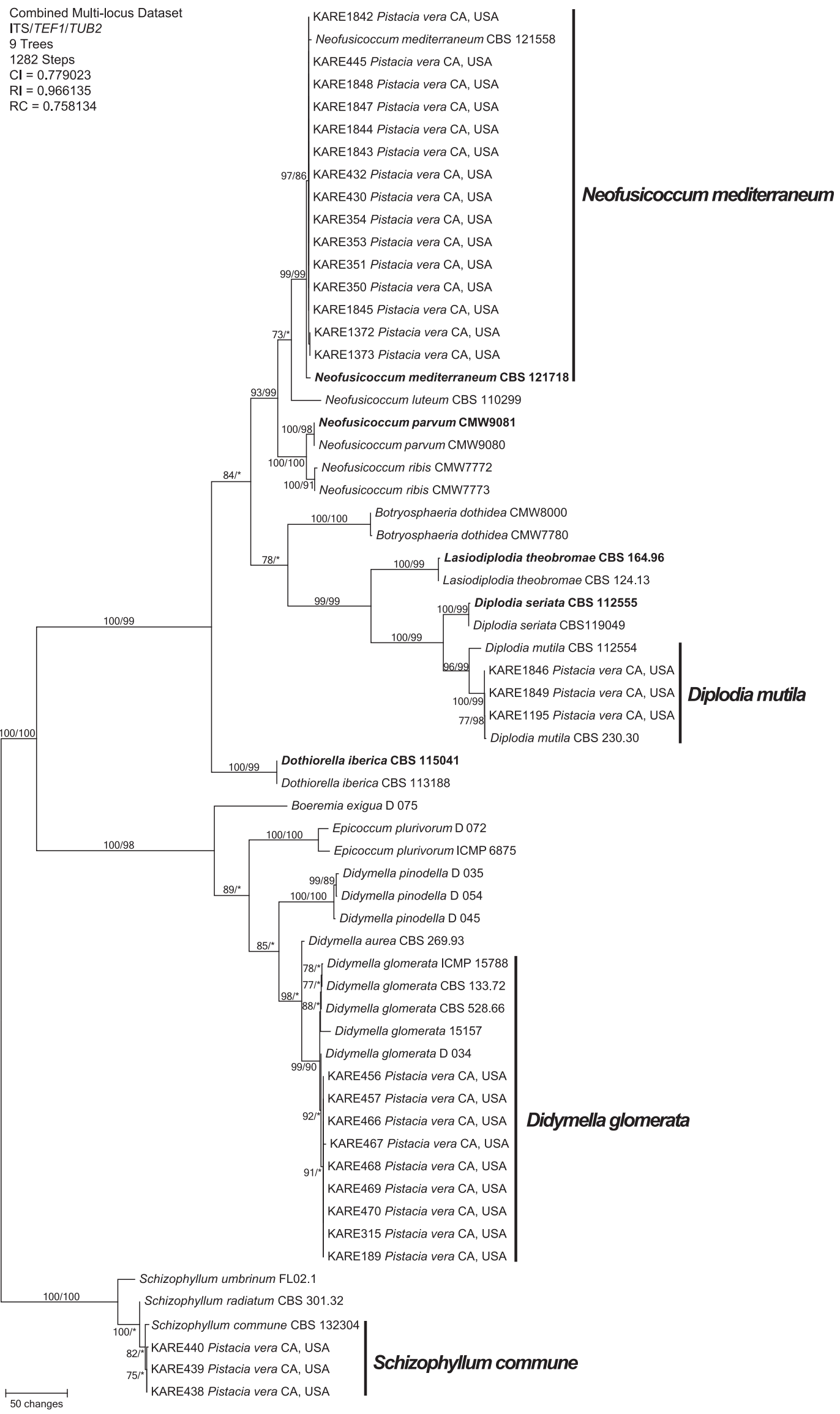

Fig. 4. One of nine equally most parsimonious trees generated from maximum parsimony analysis of the three-gene (ITS, TEF1, and TUB2) Dothideomycete combined data set. Numbers before and after the slash represent parsimony and likelihood bootstrap values from 1,000 replicates, respectively. Values with an asterisk were $<70 \%$ for the bootstrap analyses. Ex-type isolates are indicated in bold. Bar indicates the number of nucleotide changes. ITS $=$ internal transcribed spacer, TEF1 $=$ translation elongation factor $1-\alpha$, TUB2 $=$ $\beta$-tubulin, $\mathrm{Cl}=$ consistency index, $\mathrm{RI}=$ retention index, $\mathrm{RC}=$ rescaled consistency index, and KARE $=$ Kearney Agricultural Research and Extension Center. 


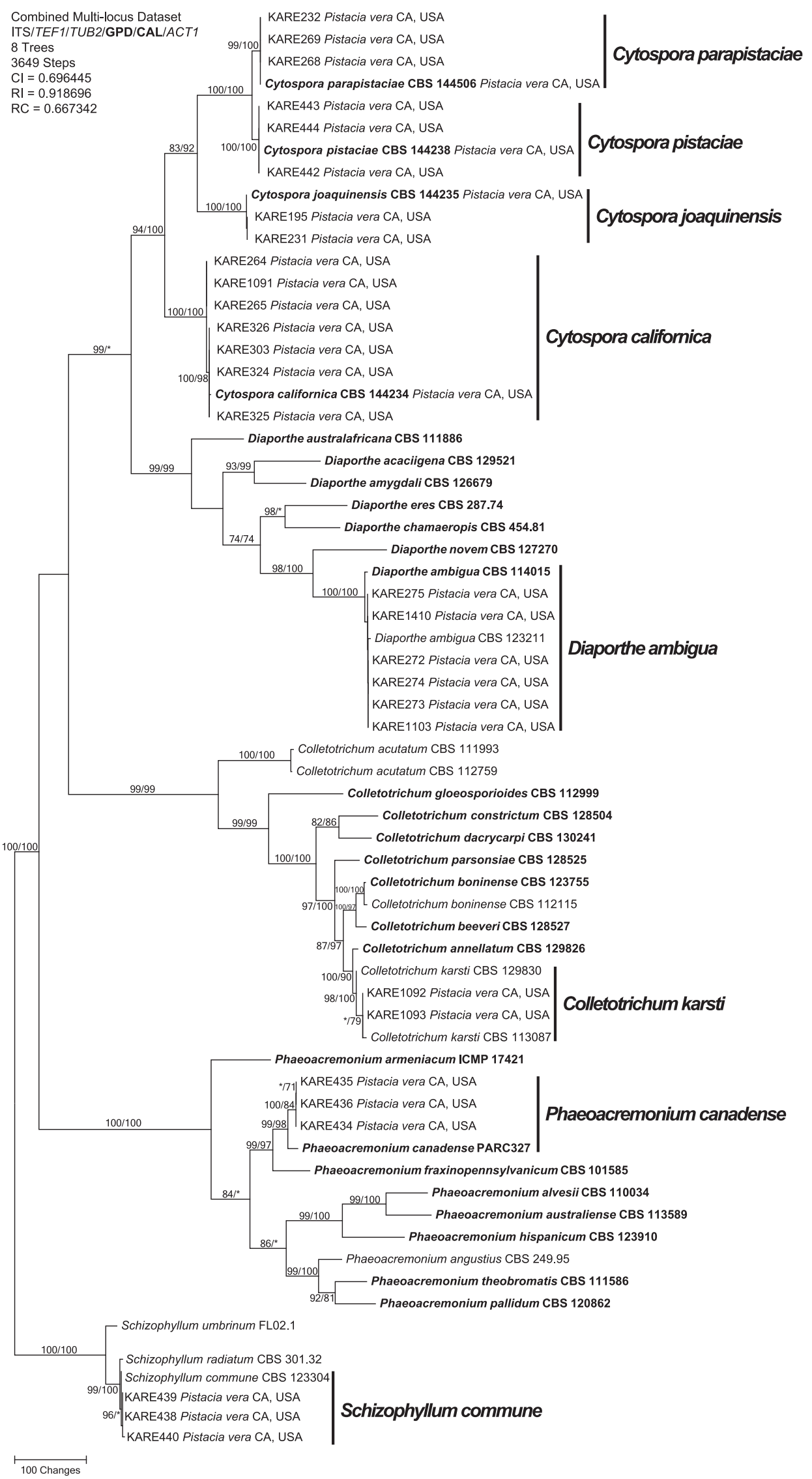

Fig. 5. One of eight equally most parsimonious trees generated from maximum parsimony analysis of the six-gene (ITS, TEF1, TUB2, GAPDH, CAL, and ACT1) Sordariomycete combined data set. Numbers before and after the slash represent parsimony and likelihood bootstrap values from 1,000 replicates, respectively. Values with an asterisk were $<70 \%$ for the bootstrap analyses. Ex-type isolates are indicated in bold. Bar indicates the number of nucleotide changes. ITS $=$ internal transcribed spacer, TEF1 = translation elongation factor 1- $\alpha, T U B 2=\beta$-tubulin, GAPDH = glyceraldehyde 3-phosphate dehydrogenase, $C A L=$ calmodulin, $A C T 1=$ actin, $\mathrm{Cl}=$ consistency index, $\mathrm{RI}=$ retention index, $\mathrm{RC}=$ rescaled consistency index, and KARE = Kearney Agricultural Research and Extension Center. 


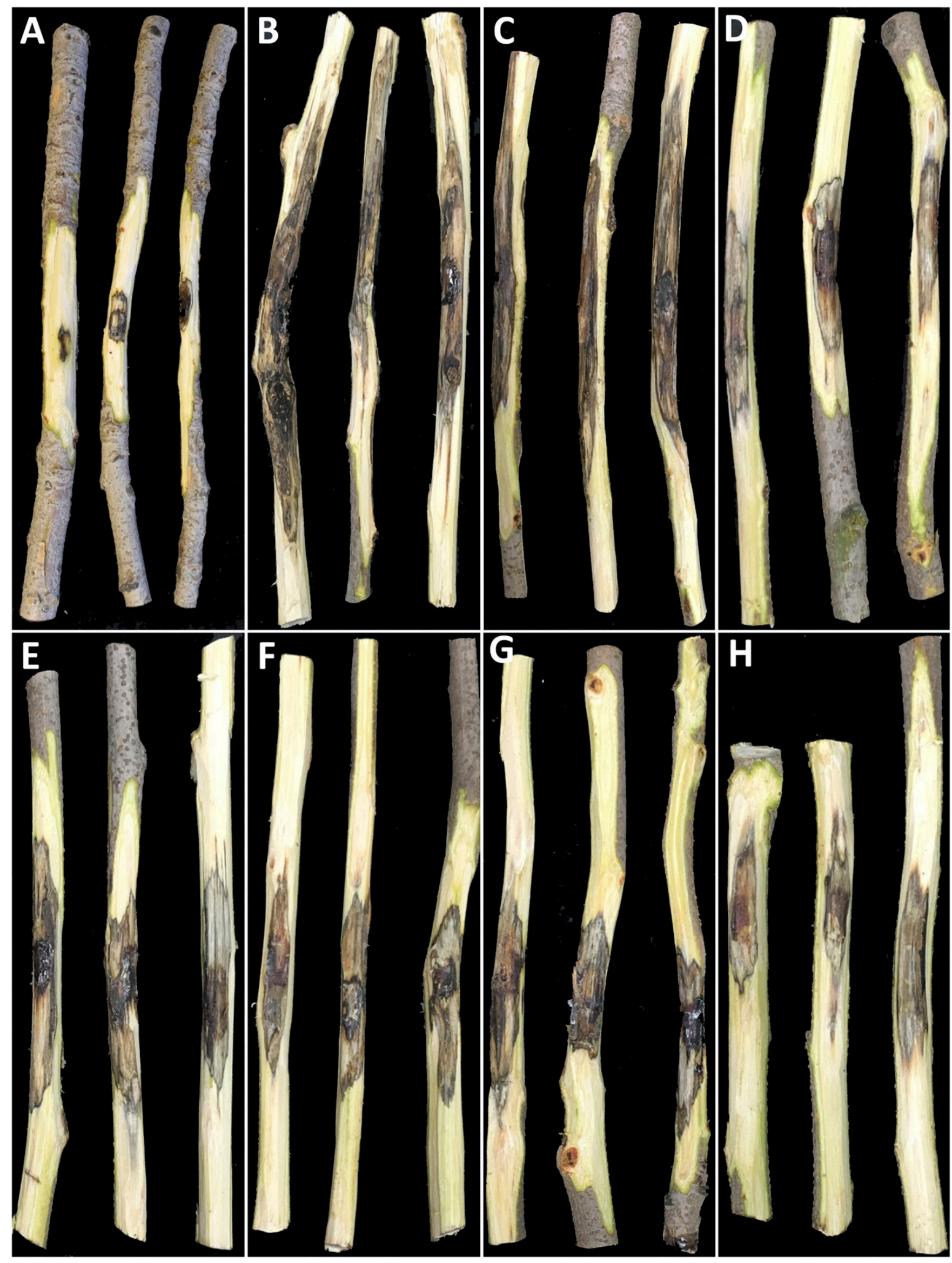

Fig. 6. Lesions produced in 2- to 3-year-old pistachio Kerman branches, 6 months after inoculation of various fungal species identified in this study: A, control, B, Neofusicoccum mediterraneum KARE350, C, Cytospora parapistaciae KARE270, D, Diaporthe ambigua KARE275, E, Didymella glomerata KARE315, F, Colletotrichum karstii KARE1092, G, Schizophyllum commune KARE440, and H, Phaeoacremonium canadense KARE435. KARE = Kearney Agricultural Research and Extension Center. 
revealed significant $(P<0.05)$ differences among the treatment means in all three cultivars (Figs. 7, 8, 9, and 10).

\section{Discussion}

This study identified 11 fungal species in eight different genera in seven different families from active cankers and associated dieback symptoms of mature pistachio trees. These included Colletotrichum karstii, Cytospora californica, Cytospora joaquinensis, Cytospora parapistaciae, Cytospora pistaciae, Diaporthe ambigua, Didymella glomerata, $N$. mediterraneum, Phaeoacremonium canadense, and $S$. commune. Pathogenicity tests using mycelium plugs showed that all fungal species can cause cankers on pistachio branches. Diplodia

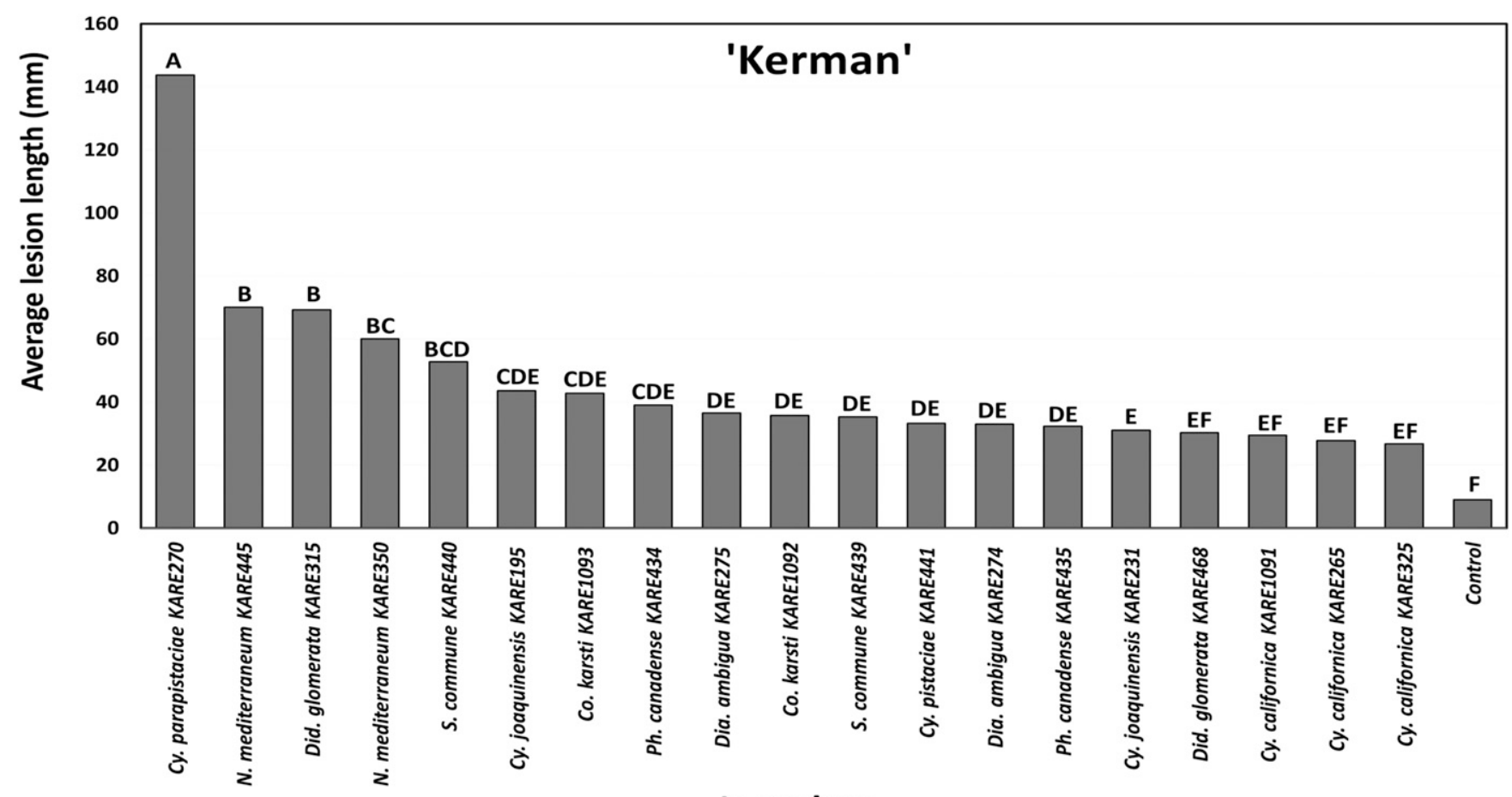

Inoculum

Fig. 7. Average lesion lengths (in millimeters) on attached Kerman pistachio branches 6 months after inoculation with mycelial plugs of 19 fungal isolates (2016 experiment). Columns with different letters indicate treatment means that are significantly different $(P<0.05)$.

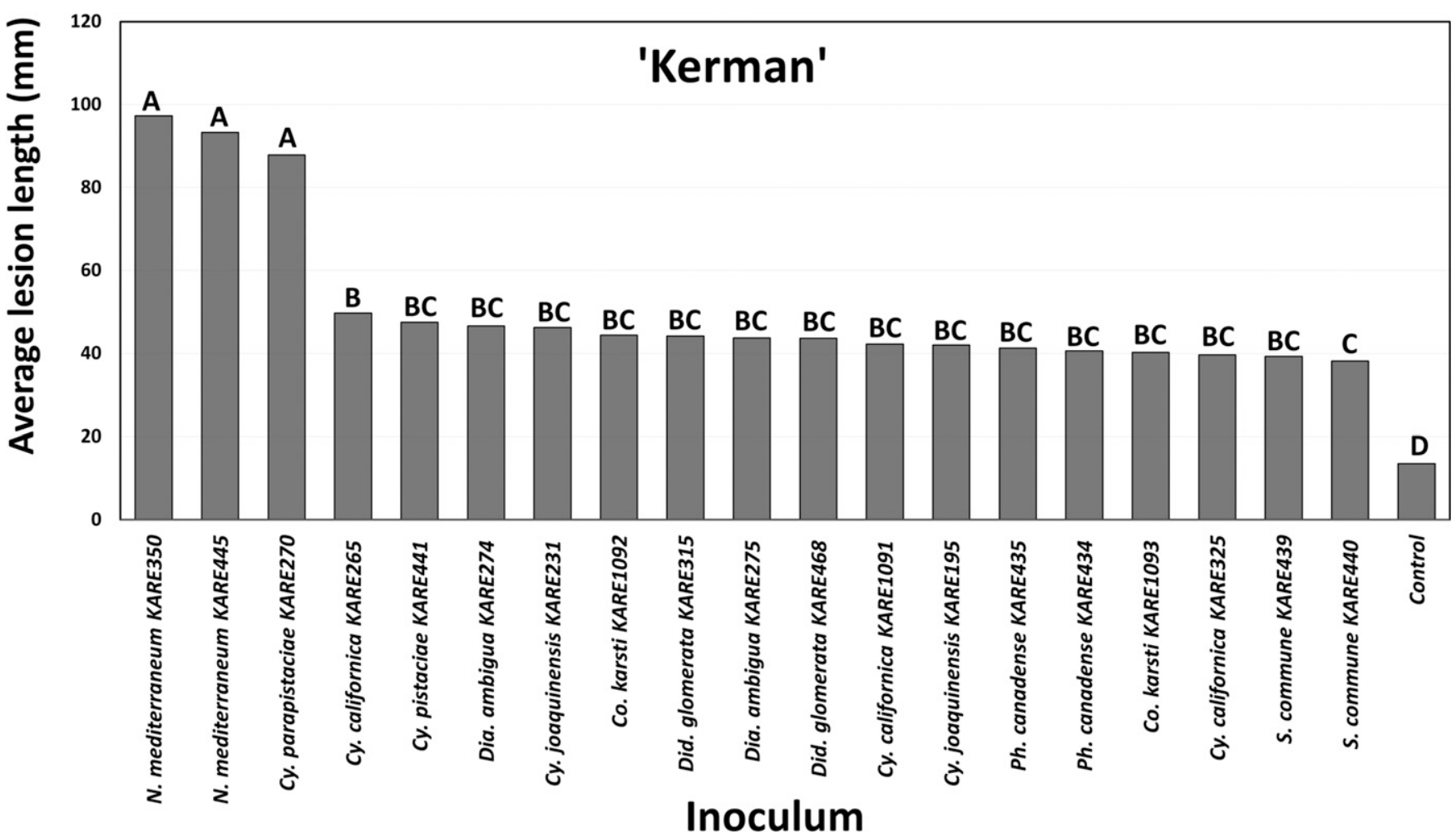

Fig. 8. Average lesion lengths (in millimeters) on attached Kerman pistachio branches 6 months after inoculation with mycelial plugs of 19 fungal isolates incubation period (2017 experiment). Columns with different letters indicate treatment means that are significantly different $(P<0.05)$. 
mutila also was recovered from pistachio cankers in Glenn County in this study; however, the pathogenicity of this species was not tested because it was acquired after the pathogenicity trials had begun.

Canker diseases in perennial crops (i.e., Malus, Prunus, and Vitis spp.) are known to cause severe yield losses by killing fruit-bearing branches and cordons that eventually lead to replantings owing to high disease severity, therefore significantly limiting the productivity and longevity of orchards and vineyards (Abe et al. 2007; Gramaje et al. 2012; Rolshausen et al. 2010). Pistachio canker and branch dieback can compromise tree canopies, resulting in the loss of fruiting

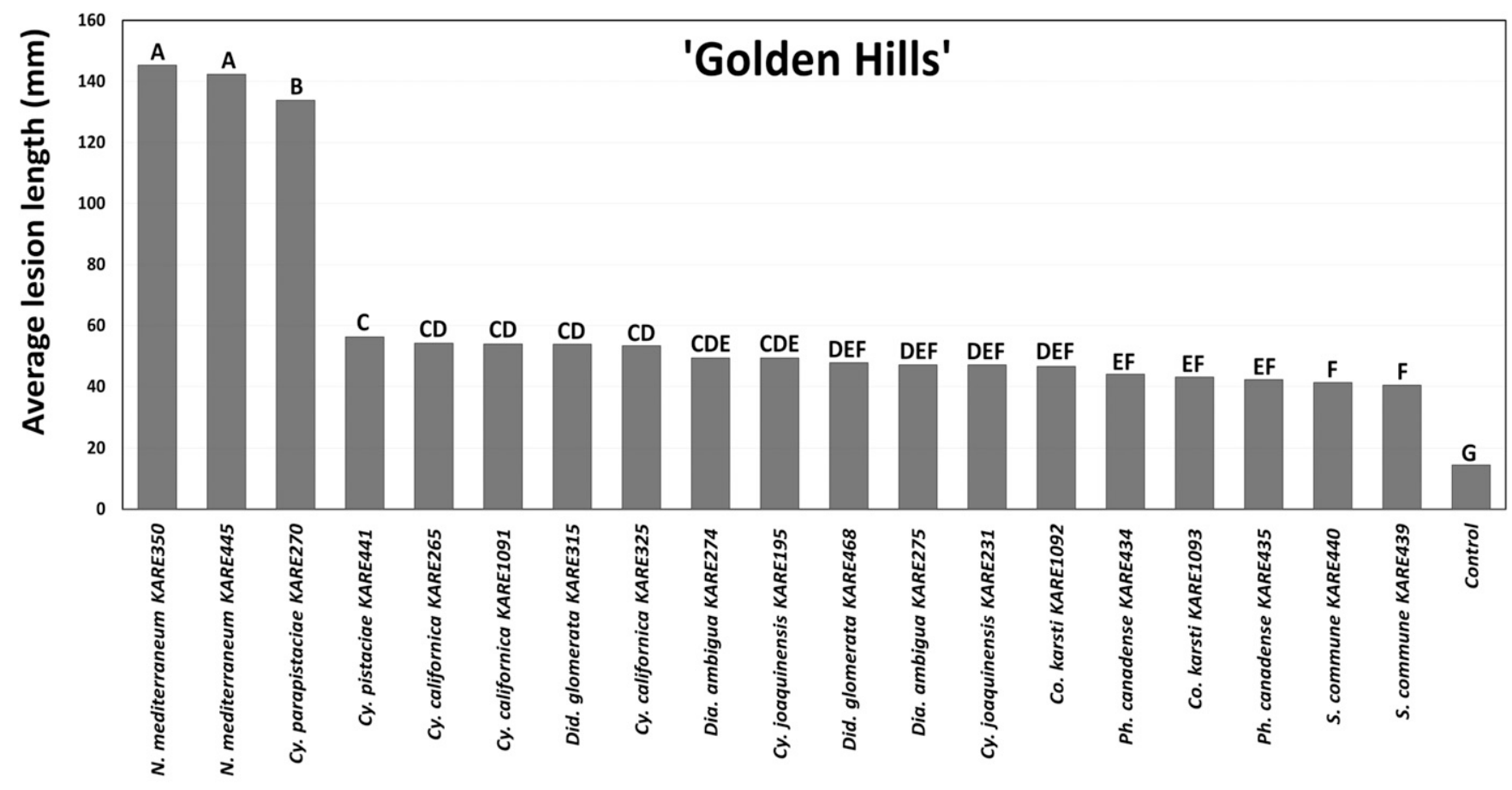

Inoculum

Fig. 9. Average lesion lengths (in millimeters) on attached Golden Hills pistachio branches 6 months after inoculation with mycelial plugs of 19 fungal isolates (2017 experiment). Columns with different letters indicate treatment means that are significantly different $(P<0.05)$.

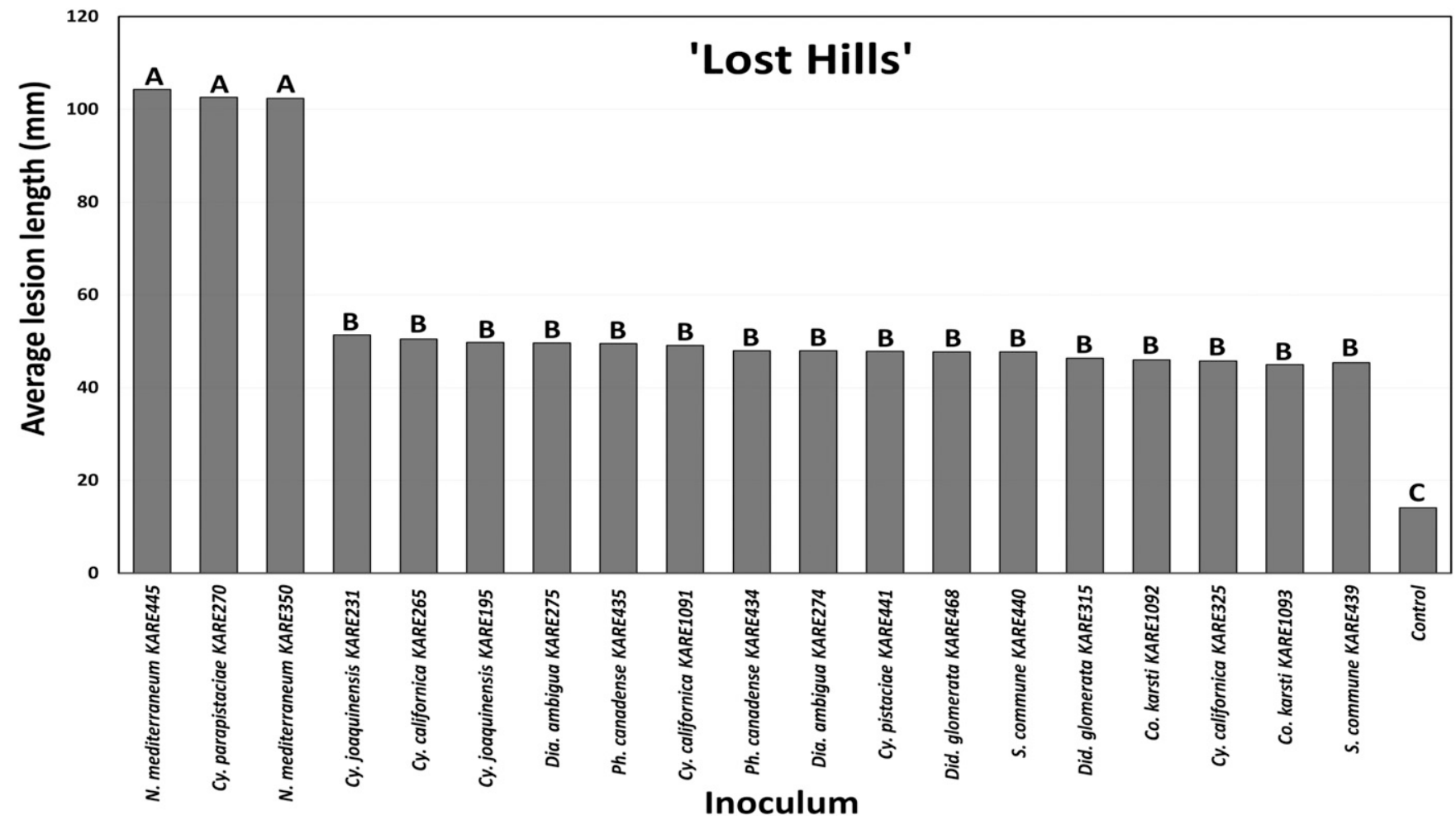

Fig. 10. Average lesion lengths (in millimeters) on attached Lost Hills pistachio branches 6 months after inoculation with mycelial plugs of 19 fungal isolates (2017 experiment). Columns with different letters indicate treatment means that are significantly different $(P<0.05)$. 
spurs. Central discoloration of wood and bark as well as necrosis of vascular tissues and dead phellogen (i.e., cambial tissue of bark) were the most frequent symptoms associated with pistachio cankers. $N$. mediterraneum-associated cankers were elongated and slightly sunken with a well-defined dark line demarcating healthy and infected tissues.

This study revealed Cytospora spp. to be the most prevalent canker pathogens of pistachio, with four species detected from symptomatic pistachio trees in California. Cytospora parapistaciae was the most aggressive pathogen on one of the most widely planted pistachio scion, Kerman, in the Central Valley, but also on Golden Hills and Lost Hills. Therefore, this species is considered an emerging threat to pistachio production in California and should be closely monitored. Currently, Cytospora parapistaciae seems to be localized to a single California County; however additional sampling will likely extend its geographical range and perhaps its host range. Other $C y$ tospora spp. detected from pistachio cankers in this study were moderately virulent. Pathogenic species of Cytospora cause substantial losses annually throughout the world to perennial crops and tree plantations, such as Prunus, Malus, Juglans, Picea, and Populus spp. (Adams et al. 2006; Fan et al. 2015; Mehrabi et al. 2011; Wang et al. 2011). The closely related Cytospora parapistaciae and Cytospora pistaciae were recovered only from pistachio in Kern and Merced counties, respectively, whereas Cytospora californica was isolated from symptomatic almond trees in Fresno, San Joaquin, and Stanislaus counties and from walnut canker in Lake County (Lawrence et al. 2018). Cytospora joaquinensis was isolated from walnut and Populus deltoides W. Bartram ex Marshall cankers in Fresno and Stanislaus counties, respectively (Lawrence et al. 2018); however, their pathogenicity to these respective hosts was not examined. Lawrence et al. (2018) suggested some level of host specificity for Cytospora parapistaciae on Pistacia vera, based on survey results and phylogenetic studies.

$N$. mediterraneum was the most prevalent species in the Botryosphaeriaceae isolated from pistachio cankers in this study, whereas Diplodia mutila was isolated from two counties only. $N$. mediterraneum is widely distributed in California perennial crops including almond, avocado, English walnut, pistachio, and olive (Chen et al. 2014a; Inderbitzin et al. 2010; Moral et al. 2010; Trouillas et al. 2010; Úrbez-Torres et al. 2013a). This study revealed that N. mediterraneum is moderately to highly virulent on Pistacia vera pistachio. N. mediterraneum is widely distributed in California perennial crops but considered only moderately virulent to almond and walnut trees (Chen et al. 2014b; Inderbitzin et al. 2010).

All other fungi tested in this study were moderately virulent to the three Pistacia vera cultivars. These fungi were also infrequently isolated and are considered of secondary importance as pistachio pathogens. One species of Diaporthe (i.e., Diaporthe ambigua) from Kern County was identified in this study. Diaporthe species may act as saprobes, endophytes, and/or plant pathogens in natural and agroecosystems (Udayanga et al. 2011). Diaporthe ambigua was reported to cause cankers on apple, pear, and plum rootstocks in South Africa (Smit et al. 1996) and it has been reported as a weak pathogen on grapevines in California (Lawrence et al. 2015; Úrbez-Torres et al. 2013b). This is the first study to test the pathogenicity of Diaporthe ambigua on three cultivars of Pistacia vera, and it was found to be moderately aggressive. Chen et al. (2014a) and Lawrence et al. (2015) reported Diaporthe chamaeropis as a mild agent of pistachio panicle and shoot blight and grapevine decline in California, respectively; however, we did not isolate Diaporthe chamaeropis in this study. A Phomopsis species causing shoot blight and leaf spot was also reported in California.

The fungus Didymella glomerata was occasionally isolated from trunk cankers of mature pistachio trees in Fresno, Kern, and Merced counties and caused lesions on pistachio branches after 6 months of incubation. Moral et al. (2018) identified Didymella glomerata as the causal agent of severe leaf blight of pistachio in Arizona. That study revealed that the pathogen did not cause symptoms on branches of pistachio 3 months after inoculation under hot and dry summer conditions, but rather suggested that the fungus is an endophyte in branches. Our pathogenicity studies were conducted in June, and branch inoculations were allowed to incubate for 6 months. Differences in the timing of inoculation and length of incubation between these different studies could explain the differences in results observed. Previously, another member of the Didymellaceae, the species Nothophoma quercina (syn. Phoma fungicola) was identified as a causal agent of stem canker and fruit blight of pistachio in Arizona (Chen et al. 2013) and dieback of olive trees in Tunisia (Taieb et al. 2014).

Additional canker pathogens isolated in low numbers in this study included Phaeoacremonium canadense in the family Togniniaceae. Phaeoacremonium spp. have been associated with Petri disease and Esca in grapevines (Gramaje et al. 2015; Mostert et al. 2006) and vascular diseases of other numerous perennial crops (Gramaje et al. 2014, 2015). Four Phaeoacremonium species (Phaeoacremonium cinereum Gramaje, Mohammadi, Banihashemi, Armengol \& L. Mostert; Phaeoacremonium minimum (Tul. \& C. Tul.) D. Gramaje, L. Mostert \& Crous; Phaeoacremonium parasiticum (Ajello, Georg \& C.J.K. Wang) W. Gams, Crous \& M.J. Wingf., and Phaeoacremonium viticola J. Dupont) were reported as canker pathogens of pistachio in Iran (Mohammadi et al. 2015); however, we did not isolate any of those species in California. Úrbez-Torres et al. (2014) revealed Phaeoacremonium canadense to be a moderate pathogen of detached canes of cultivar Chardonnay and potted rootstocks of cultivar 3309C grapevines in British Columbia, Canada. The pathogenicity results of Úrbez-Torres et al. (2014) and ours suggest that this fungus is weakly to moderately virulent to both grapevine and pistachio.

The basidiomycete $S$. commune that is involved in wood decay on a number of fruit and nut trees (Fischer and Gonzalez-Garcia 2015) was found occasionally in cankers of pistachio trees in this study. This suggests pruning-wound colonization (Snieškienè and Juronis 2001) by basidiospores from nearby basidiocarps fruiting on dead branches (Shimizu et al. 2008). On grapevine, S. commune is reported as usually growing saprophytically in dead bark and less frequently in living wood (Fischer and Gonzalez-Garcia 2015). Inoculations in the present study indicated that this species is moderately virulent to pistachio in California. The fungus was reported as a weak pathogen on olive trees in California (Úrbez-Torres et al. 2013a).

Colletotrichum karstii was the least common fungus isolated from pistachio cankers in this study. Colletotrichum is one of the most economically important pathogenic genera, causing anthracnose of fruits and leaves and affecting a wide range of hosts in tropical and subtropical regions (Cannon et al. 2012; Damm et al. 2009). Various Colletotrichum species are known to produce anthracnose of pistachio in Australia, China, and California (Ash and Lanoiselet 2001; Lichtemberg et al. 2017; Yang et al. 2011). The occurrence of Colletotrichum karstii in cankers of pistachio tree has not been reported previously, and the role of this fungus as a canker pathogen is poorly understood.

In conclusion, this work represents the first detailed study on the identity and pathogenicity of fungal species associated with canker diseases of pistachio in California. Eleven species were isolated and pathogenicity tests indicated that all were moderately to highly virulent to Kerman, Golden Hills, and Lost Hills pistachio. Most of the identified species have been reported from other fruit and nut trees; frequently, these different tree species are adjacent or in close proximity to pistachio orchards. This suggests that cross-infection may occur among these crops. Although they are relatively infrequent in pistachio orchards $<15$ years old, canker diseases must be monitored carefully in pistachio and other perennial crops. Many studies have shown that perennial crops subjected to stress become more susceptible to infection by canker-causing fungal pathogens (Colhoun 1973; Desprez-Loustau et al. 2006; Ma et al. 2001). Canker diseases in almond and walnut orchards and vineyards in California have been of increasing concern in the last 10 years (Chen et al. 2014b; Nouri et al. 2018; Úrbez-Torres et al. 2010, 2013b). Specific control measures are being investigated in these crops to mitigate the impact of canker diseases. Future research on disease management of pistachio canker diseases should focus on these most widespread and virulent pathogens reported here. 


\section{Acknowledgments}

We thank Dr. Themis Michailides for providing isolates for this study.

\section{Literature Cited}

Abe, K., Kotoda, N., Kato, H., and Soejima, J. 2007. Resistance sources to Valsa canker (Valsa ceratosperma) in a germplasm collection of diverse Malus species. Plant Breed. 126:449-453.

Adams, G. C., Roux, J., and Wingfield, M. J. 2006. Cytospora species (Ascomycota, Diaporthales, Valsaceae): Introduced and native pathogens of trees in South Africa. Australas. Plant Pathol. 35:521-548.

Adams, G. C., Wingfield, M. J., Common, R., and Roux, J. 2005. Phylogenetic relationships and morphology of Cytospora species and related teleomorphs (Ascomycota, Diaporthales, Valsaceae) from Eucalyptus. Stud. Mycol. 52:1-144.

AL-Saghir, M. G., and Porter, D. M. 2012. Taxonomic revision of the genus Pistacia L. (Anacardiaceae). Am. J. Plant Sci. 3:12-32.

Armengol, J., Gramaje, D., Pérez-Sierra, A., Landeras, E., Alzugaray, R., Martos, S., and Luque, J. 2008. First report of canker disease caused by Neofusicoccum australe on eucalyptus and pistachio in Spain. Plant Dis. 92:980.

Ash, G. J., and Lanoiselet, V. M. 2001. First report of Colletotrichum acutatum causing a leaf spot and hull rot of pistachio. Australas. Plant Pathol. 30:365-366.

Ashworth, L. J., Jr., Morgan, D. P., and Surber, E. 1986. Verticillium wilt of pistachio. Calif. Agric. 21-24.

Bertrand, P., and English, H. 1976. Virulence and seasonal activity of Cytospora leucostoma and Cytospora cincta in French prune trees in California. Plant Dis. Rep. 60:106-110.

Cannon, P. F., Damm, U., Johnston, P. R., and Weir, B. S. 2012. Colletotrichum current status and future directions. Stud. Mycol. 73:181-213.

Carbone, I., and Kohn, L. M. 1999. A method for designing primer sets for speciation studies in filamentous ascomycetes. Mycologia 91:553-556.

Chen, S. F., Li, G. Q., Liu, F. F., and Michailides, T. J. 2015. Novel species of Botryosphaeriaceae associated with shoot blight of pistachio. Mycologia 107: 780-792.

Chen, S. F., Morgan, D. P., Hasey, J. K., Anderson, K., and Michailides, T. J. 2014b. Phylogeny, morphology, distribution, and pathogenicity of Botryosphaeriaceae and Diaporthaceae from English walnut in California. Plant Dis. 98:636-652.

Chen, S. F., Morgan, D. P., and Michailides, T. J. 2013. First report of Phoma fungicola associated with stem canker and fruit blight of pistachio in Arizona. J. Plant Pathol. 95:451.

Chen, S. F., Morgan, D. P., and Michailides, T. J. 2014a. Botryosphaeriaceae and Diaporthaceae associated with panicle and shoot blight pistachio in California, USA. Fungal Divers. 67:157-179.

Colhoun, J. 1973. Effects of environmental factors on plant disease. Annu. Rev. Phytopathol. 11:343-364.

Damm, U., Cannon, P. F., Woudenberg, J. H. C., Johnston, P. R., Weir, B. S., Tan, Y. P., Shivas, R. G., and Crous, P. W. 2012. The Colletotrichum boninense species complex. Stud. Mycol. 73:1-36.

Damm, U., Mostert, L., Crous, P. W., and Fourie, P. H. 2008. Novel Phaeoacremonium species associated with necrotic wood of Prunus trees. Persoonia 20:87-102.

Damm, U., Woudenberg, J. H. C., Cannon, P. F., and Crous, P. W. 2009. Colletotrichum species with curved conidia from herbaceous hosts. Fungal Divers. 39:45-87.

Desprez-Loustau, M. L., Marcais, B., Nageleisen, L. M., Piou, D., and Vannini, A. 2006. Interactive effects of drought and pathogens in forest trees. Ann. Sci. 63: 597-612.

Fan, X., Hyde, K. D., Liu, M., Liang, Y., and Tian, C. 2015. Cytospora species associated with walnut canker disease in China, with description of a new species C. gigalocus. Fungal Biol. 119:310-319.

Farr, D. F., and Rossman, A. Y. 2015. U.S. Department of Agriculture Agricultural Research Service Fungal Databases. https://nt.ars-grin.gov/ fungaldatabases/

Fischer, M., and Gonzalez-Garcia, V. 2015. An annotated checklist of European basidiomycetes related to white rot of grapevine (Vitis vinifera). Phytopathol. Mediterr. 54:281-298.

French, A. M. 1989. California Plant Disease Host Index. California Department of Food and Agriculture Division of Plant Industry, Sacramento.

Glass, N. L., and Donaldson, G. C. 1995. Development of primer sets designed for use with the PCR to amplify conserved genes from filamentous Ascomycetes. Appl. Environ. Microbiol. 61:1323-1330.

Gramaje, D., Agustí-Brisach, C., Pérez-Sierra, A., Moralejo, E., Olmo, D., Mostert, L., Damm, U., and Armengol, J. 2012. Fungal trunk pathogens associated with wood decay of almond trees on Mallorca (Spain). Persoonia 28:1-13.

Gramaje, D., León, M., Pérez-Sierra, A., Burgess, T., and Armengol, J. 2014. New Phaeoacremonium species isolated from sandalwood trees in Western Australia. IMA Fungus 5:67-77.

Gramaje, D., Mostert, L., Groenewald, J. Z., and Crous, P. W. 2015. Phaeoacremonium: From esca disease to phaeohyphomycosis. Fungal Biol. 119:759-783.

Guerber, J. C., Liu, B., Johnston, P., and Correll, J. C. 2003. Characterization of diversity in Colletotrichum acutatum sensu lato by sequence analysis of two introns, mtDNA and intron RFLPs, and mating compatibility. Mycologia 95: 872-895.

Inderbitzin, P., Bostock, R. M., Trouillas, F. P., and Michailides, T. J. 2010. A six locus phylogeny reveals high species diversity in Botryosphaeriaceae from California almond. Mycologia 102:1350-1368.

Krid Hadj Taieb, S., Triki, M. A., Hammami, I., and Rhouma, A. 2014. First report of dieback of olive trees caused by Phoma fungicola in Tunisia. J. Plant Pathol. 96(suppl):4.117.

Lawrence, D. P., Holland, L. A., Nouri, M. T., Travadon, R., Abramians, A. Michailides, T. J., and Trouillas, F. P. 2018. Molecular phylogeny of Cytospora species associated with canker diseases of fruit and nut crops in California, with the descriptions of ten new species and one new combination. IMA Fungus 9:333-370.

Lawrence, D. P., Travadon, R., and Baumgartner, K. 2015. Diversity of Diaporthe species associated with wood cankers of fruit and nut crops in northern California. Mycologia 107:926-940.

Lawrence, D. P., Travadon, R., Nita, M., and Baumgartner, K. 2017b. TrunkDiseaseID.org: A molecular database for fast and accurate identification of fungi commonly isolated from grapevine wood. Crop Prot. 102:110-117.

Lawrence, D. P., Travadon, R., Pouzoulet, J., Rolshausen, P., Wilcox, W., and Baumgartner, K. 2017a. Characterization of Cytospora isolates from wood cankers of declining grapevine in North America, with the descriptions of two new Cytospora species. Plant Pathol. 66:713-725.

Lichtemberg, P. S. F., Moral, J., Morgan, D. P., Felts, D. G., Sanders, R. D., and Michailides, T. J. 2017. First report of anthracnose caused by Colletotrichum fioriniae and C. karstii in California pistachio orchards. Plant Dis. 101:1320.

Linaldeddu, B. T., Maddau, L., Franceschini, A., Alves, A., and Phillips, A. J. L. 2016. Botryosphaeriaceae species associated with lentisk dieback in Italy and description of Diplodia insularis sp. nov. Mycosphere. 7:962-977.

Ma, Z., Morgan, D. P., and Michailides, T. J. 2001. Effects of water stress on Botryosphaeria blight of pistachio caused by Botryosphaeria dothidea. Plant Dis. 85:745-749.

Maddison, W. P., and Maddison, D. R. 2016. Mesquite: A modular system for evolutionary analysis, version 3.10. http://www.mesquiteproject.org

Mehrabi, M. E., Mohammadi, G. E., and Fotouhifar, K. B. 2011. Studies on Cytospora canker disease of apple trees in Semirom region of Iran. J. Agric. Technol. 7:967-982.

Michailides, T. J. 1991. Pathogenicity, distribution, sources of inoculum, and infection courts of Botryosphaeria dothidea on pistachio. Phytopathology 81:566-573.

Michailides, T. J. 2005. Pest, disease, and physiological disorders management: Above ground fungal diseases. http://fruitsandnuts.ucdavis.edu/files/73707.pdf

Michailides, T. J., Morgan, D. P., and Felts, D. 2002. First report of Botryosphaeria rhodina causing shoot blight of pistachio in California. Plant Dis. $86: 1273$.

Mohammadi, H., Sarcheshmehpour, M., and Mafi, E. 2015. Fungal trunk pathogens associated with wood decay of pistachio trees in Iran. Span. J. Agric. Res. 13:e1007.

Moral, J., Lichtemberg, P. S. F., Papagelis, A., Sherman, J., and Michailides, T. J. 2018. Didymella glomerata causing leaf blight on pistachio. Eur. J. Plant Pathol. 151:1095-1099.

Moral, J., Muñoz-Díez, C., González, N., Trapero, A., and Michailides, T. J. 2010. Characterization and pathogenicity of Botryosphaeriaceae species collected from olives and other hosts in Spain and California. Phytopathology 100: 1340-1351.

Mostert, L., Groenewald, J. Z., Gams, W., Summerbell, R. C., and Crous, P. W. 2006. Taxonomy and pathology of Togninia (Diaporthales) and its Phaeoacremonium anamorphs. Stud. Mycol. 54:1-113.

Mostert, L., Groenewald, J. Z., Summerbell, R. C., Robert, V., Sutton, D. A., Padhye, A. A., and Crous, P. W. 2005. Species of Phaeoacremonium associated with human infections and environmental reservoirs in infected woody plants. J. Clin. Microbiol. 43:1752-1767.

Nouri, M. T., Lawrence, D. P., Yaghmour, M. A., Michailides, T. J., and Trouillas, F. P. 2018. Neoscytalidium dimidiatum causing canker, shoot blight and fruit rot of almond in California. Plant Dis. 102:1638-1647.

O’Donnell, K., and Cigelnik, E. 1997. Two divergent intragenomic rDNA ITS2 types within a monophyletic lineage of the fungus Fusarium are nonorthologous. Mol. Phylogenet. Evol. 7:103-116.

Pryor, B. M., and Michailides, T. J. 2002. Morphological, pathogenic, and molecular characterization of Alternaria isolates associated with Alternaria late blight of pistachio. Phytopathology 92:406-416.

Rolshausen, P. E., Úrbez-Torres, J. R., Rooney-Latham, S., Eskalen, A., Smith, R. J., and Gubler, W. D. 2010. Evaluation of pruning wound susceptibility and protection against fungi associated with grapevine trunk diseases. Am. J. Enol. Vitic. 61:113-119.

Shimizu, J., Hayashi, Y., and Fukuda, K. 2008. Wood-rot disease on cherry trees along Koganei Cherry Street, a national cultural property. Landsc. Res. J. 71: 865-868 [In Japanese with English summary].

Smit, W. A., Viljoen, C. D., Wingfield, B. D., Wingfield, M. J., and Calitz, F. J. 1996. A new canker disease of apple, pear and plum rootstocks caused by Diaporthe ambigua in South Africa. Plant Dis. 80:1331-1335.

Snieškiene, V., and Juronis, V. 2001. Distribution of the fungus Schizophyllum commune Fr. in plantings of trees in the Kaunas city. Biologija (Vilnius) 3: $45-47$ 
Swart, W. J., and Blodgett, J. T. 1998. First report of Botryosphaeria dothidea basal canker of pistachio trees in South Africa. Plant Dis. 82:960.

Tamura, K., Stecher, G., Peterson, D., Filipski, A., and Kumar, S. 2013. MEGA6: Molecular evolutionary genetics analysis version 6.0. Mol. Biol. Evol. 30:2725-2729.

Trouillas, F. P., Peduto, F., Lorber, J., Sosnowski, M., Grant, J., Coates, W., Anderson, K., Caprile, J., and Gubler, W. D. 2012. Calosphaeria canker of sweet cherry caused by Calosphaeria pulchella in California and South Australia. Plant Dis. 96:648-658.

Trouillas, F. P., Úrbez-Torres, J. R., Peduto, F., and Gubler, W. D. 2010. First report of twig and branch dieback of English walnut (Juglans regia) caused by Neofusicoccum mediterraneum in California. Plant Dis. 94:1267.

Udayanga, D., Liu, X., McKenzie, E. H. C., Chukeatirote, E., Bahkali, A. H. A., and Hyde, K. D. 2011. The genus Phomopsis: Biology, applications, species concepts and names of common phytopathogens. Fungal Divers. 50:189-225.

Úrbez-Torres, J. R., Haag, P., Bowen, P., and O'Gorman, D. T. 2014. Grapevine trunk diseases in British Columbia: Incidence and characterisation of the fungal pathogens associated with esca and Petri diseases of grapevine. Plant Dis. 98: 469-482.

Úrbez-Torres, J. R., Peduto, F., and Gubler, W. D. 2010. First report of grapevine cankers caused by Lasiodiplodia crassispora and Neofusicoccum mediterraneum in California. Plant Dis. 94:785.
Urbez-Torres, J. R., Peduto, F., Smith, R., and Gubler, W. D. 2013b. Phomopsis dieback: A grapevine trunk disease caused by Phomopsis viticola in California. Plant Dis. 97:1571-1579.

Úrbez-Torres, J. R., Peduto, F., Vossen, P. M., Krueger, W. H., and Gubler, W. D 2013a. Olive twig and branch dieback: Etiology, incidence, and distribution in California. Plant Dis. 97:231-244.

Vitale, S., Aiello, D., Guarnaccia, V., Luongo, L., Galli, M., Crous, P. W., Polizzi, G., Belisario, A., and Voglmayr, H. 2018. Liberomyces pistaciae sp. nov., the causal agent of pistachio cankers and decline in Italy. MycoKeys 40:29-51.

Wang, X., Wei, J., Huang, L., and Kang, Z. 2011. Re-evaluation of pathogens causing Valsa canker on apple in China. Mycologia 103:317-324.

White, T. J., Bruns, T., Lee, S., and Taylor, J. 1990. Amplification and direct sequencing of fungal ribosomal RNA genes for phylogenetics. Pages 315-322 in: PCR Protocols: A Guide to Methods and Applications. M. A. Innis, D. H. Gelfand, J. J. Snisky, and T. J. White, eds. Academic Press, San Diego, CA.

Wunderlich, N., Costa, S. S., Tpoi, R. P., and Ash, G. J. 2012. First report of Botryosphaeria dothidea causing shoot blight and cankers of pistachio in Australia. Australas. Plant Dis. Notes 7:47-49.

Yang, S. Y., Su, S. C., Liu, T., Fan, G., and Wang, J. 2011. First report of anthracnose caused by Colletotrichum gloeosporioides on pistachio (Pistacia vera) in China. Plant Dis. 95:1314. 